Claremont Colleges

Scholarship@ Claremont

All HMC Faculty Publications and Research

HMC Faculty Scholarship

$12-1-1988$

\title{
Quasidegenerate Variational Perturbation Theory and the Calculation of First-Order Properties from Variational Perturbation Theory Wave Functions
}

\author{
Robert J. Cave \\ Harvey Mudd College \\ Ernest R. Davidson \\ Indiana University - Bloomington
}

\section{Recommended Citation}

Quasidegenerate variational perturbation theory and the calculation of first-order properties from variational perturbation theory wave functions. Robert J. Cave and Ernest R. Davidson, J. Chem. Phys. 89, 6798 (1988), DOI: 10.1063/1.455354

This Article is brought to you for free and open access by the HMC Faculty Scholarship at Scholarship @ Claremont. It has been accepted for inclusion in All HMC Faculty Publications and Research by an authorized administrator of Scholarship @ Claremont. For more information, please contact scholarship@cuc.claremont.edu. 


\title{
Quasidegenerate variational perturbation theory and the calculation of first- order properties from variational perturbation theory wave functions
}

\author{
Robert J. Cave and Ernest R. Davidson a) \\ Department of Chemistry, Indiana University, Bloomington, Indiana 47405
}

(Received 18 July 1988; accepted 16 August 1988)

\begin{abstract}
In previous work on the treatment of correlation in molecular systems we have applied a multireference version of second-order Hylleraas variational perturbation theory. The choice made for the partitioning of $H$ treated the interactions between the correlating functions to infinite order and gave the corrections to the wave function to first order. The method was shown to be accurate in many cases, but became less so when near degeneracies occurred between the reference energy and other eigenvalues of $H_{0}$. In this article we introduce an effective Hamiltonian method that is analogous to variational perturbation theory, but which is significantly more accurate when near degeneracies are important. This quasidegenerate variational perturbation theory (QDVPT) is an explicitly multireference procedure and treats the entire reference space as a quasidegenerate space. A novel method for solving the QDVPT equations is introduced that avoids explicit construction of the effective Hamiltonian. As a result, the work involved in application of QDVPT is on the roder of that required for variational perturbation theory. We also present an approximate method for calculating firstorder atomic and molecular properties based on Hylleraas variational perturbation theory, multireference linearized coupled cluster, and QDVPT wave functions. The properties are calculated as derivatives of the energy with respect to the field strength. Construction of a oneelectron density matrix based on the energy derivative expression allows rapid evaluation of one-electron properties. Results are presented and compared to full and truncated CI results. Good agreement is found in the cases examined.
\end{abstract}

\section{INTRODUCTION}

One of the goals in the development of ab initio quantum chemical methods is to obtain procedures which yield sizeconsistent (or size-extensive, we use the two terms interchangeably here) results. 'A size-consistent method is one in which the energy of the system scales linearly with the number of particles. Some examples of size-consistent methods are restricted and unrestricted Hartree-Fock theory, Moller-Plesset perturbation theory, some types of completeactive-space SCF, coupled-cluster theory, and full configuration interaction calculations. Truncated configuration interaction $(\mathrm{CI})$ is an example of a size-inconsistent method; for a singles and doubles $\mathrm{CI}$ the correlation energy scales as $\checkmark n$, where $n$ is the number of particles. However, it has been shown in systems containing only a few electrons and a small basis set that multireference singles and doubles $\mathrm{CI}$ (MRSDCI) results can accurately reproduce full CI results. ${ }^{2}$ Comparisons have not been made for larger systems because the full CIs cannot be performed at the present time. For treatment of large systems it will be imperative to develop methods that are more nearly size-consistent than singlereference based SDCI.

Until recently, the predominant size-consistent correlation methods (Møller-Plesset perturbation theory and coupled-cluster theory) were applied almost exclusively as sin-

"Author to whom correspondence should be addressed. gle-reference based procedures. In part this is due to the added computational complexity of the multireference based procedures, but also because of the rather severe requirements on the reference space in the early formulations of multireference theories. ${ }^{3}$ With large reference spaces problems arose due to the presence of intruder states, giving rise to numerical instabilities or poor convergence of the perturbation series. ${ }^{4}$ In addition, it has been shown that in many cases single-reference based procedures can be quite robust and can overcome the rather severe limitations imposed by restriction to a single reference function. In a study by Laidig et al. ${ }^{5}$ it was shown that a coupled-cluster model including single, double, and triple excitations was able to describe the potential curve for breaking a single bond with quite high accuracy.

However, a host of problems exist for which a singleconfigurational description is inappropriate. Excited states of the same symmetry as the ground state, or higher lying states of other symmetries than the ground state are cases where adequate single-configurational descriptions may be difficult to obtain. In addition, points on the ground state surface may be inherently multiconfigurational, such as some transition states. It is therefore desirable to have multiconfigurational methods that are size consistent. The earliest applications of multireference coupled-cluster theory ${ }^{5-12}$ were all based on complete-active-space SCF reference spaces. In previous applications of multireference coupledcluster theory, the coupled-cluster equations have been truncated at second order to yield manageable calculations. In 
most cases, ${ }^{5,6,9-11}$ the orthogonal complement of the reference space was excluded from the expansion of the wave functions. When this approximation is made one is dependent on the complete-active-space SCF to yield an accurate estimate of the relative weights of the configurations in the refrence space. In more recent developments the requirement of a complete-active-space SCF reference space has been relaxed. ${ }^{13,14} \mathrm{~A}$ method related to multireference coupled-cluster approaches is the multireference averaged coupled-pair-functional approach of Gdanitz and Ahlrichs. ${ }^{15}$ In this method the orthogonal complement of the reference wave function is included in the wave function expansion. While not strictly size consistent, it has been shown to be quite accurate for a variety of small- and moderate-sized systems. ${ }^{15}$

We have recently proposed a version of variational perturbation theory ${ }^{16-18}$ based on a multireference zeroth-order space. It was shown to differ from the multireference linearized coupled-cluster ${ }^{5-7}$ (MRLCC) method in that the orthogonal complement of the reference space was included in the wave function expansion. Thus, variational perturbation theory allows correlation effects to alter the relative weights of the zeroth-order configurations in the final wave function. In test calculations ${ }^{18}$ variational perturbation theory (VPT) gave excitation energies and total energies in good agreement with MRLCC and full CI results. However, in further testing of the method we have found that the inclusion of the orthogonal complement functions in the wave function can make the method more susceptible to intruder state effects than, e.g., MRLCC. In Sec. II we discuss the origin of this effect and introduce a simple model to illustrate the problem. It will be shown that this effect is a result of the coupling of the orthogonal complement of the zeroth-order wave function to all single and double excitations. In Sec. V computational results on this model will be presented to support ehe qualitative discussion of Sec. II.

In many cases the intruder state problem in VPT does not manifest itself, but when it does one may be unwilling to merely remove the orthogonal complement functions from the wave function and use MRLCC theory. In. Sec. III we present an effective Hamiltonian based analog of VPT, which we call quasidegenerate variational perturbation theory (QDVPT). Quasidegenerate variational perturbation theory treats the reference wave function $\left(\Psi_{0}\right)$ and its orthogonal complement on a more equal basis. The partitioning made of $H$ into $H_{0}$ and $V$ is similar to that made in VPT, ${ }^{18}$ except that the reference space (P) now contains $\Psi_{0}$ and its orthogonal complement, while the correlation space $(\mathbf{Q})$ only contains the single and double excitations relative to $\mathbf{P}$. We retain the full coupling of the single and double excitations between themselves in $H_{0}$, thus yielding an infinite order treatment of their interactions as in VPT. In the limit that the effective interaction within the reference space goes to zero, QDVPT yields results identical with MRLCC. When the effective coupling is small but nonzero QDVPT gives results similar to VPT. However, where intruder state problems arise is variational perturbation theory, or where correlation brings on significant reference space readjustments, results indicate that QDVPT is superior to VPT.
QDVPT will be shown to be not strictly size consistent, but to be much more nearly so than (MRSDCI).

In Sec. IV we introduce a method for estimating firstorder one-electron properties based on VPT and QDVPT wave functions. Not only do the one-electron properties provide additional probes of size-inconsistency effects, but it is sometimes the case that one-electron properties are of more interest in a theoretical study than bond energies or excitation energies. Also, comparison of calculated molecular properties with experimental values can provide a means of gauging the quality of a calculational procedure. ${ }^{19}$ It is thus useful to be able to evaluate molecular properties in an accurate and efficient manner. This is particularly true for correlated wave functions, where one has some hope of systematically approaching agreement with experimental quantities.

The Hellmann-Feynman theorem ${ }^{20}$ states that for the exact wave function one can obtain equivalent results for first-order properties by calculating the properties as derivatives of the energy with respect to the perturbation of interest, or as expectation values of the perturbation over the unperturbed wave function. This is also true for wave functions that are optimal with respect to all variable parameters, such as SCF, complete-active-space multiconfigurational $\mathrm{SCF}$, and full configuration interaction. ${ }^{21}$ Most other commonly employed wave functions (truncated configuration interaction, ${ }^{22}$ many-body perturbation theory, ${ }^{22}$ coupledcluster approaches,' coupled-pair functional theory, ${ }^{15}$ and variational perturbation theory ${ }^{16-18}$ ) are not optimal with respect to all parameters and differences are expected between the energy derivative and expectation value methods of property evaluation.

The disadvantage of the energy-derivative methods applied in a a finite-field form ${ }^{23}$ is that several calculations of the wave function must be performed for each property desired. The expectation value method, where a reduced oneparticle density matrix can be defined, allows efficient evaluation of many properties at once. However, for some correlated wave functions in current use (most notably coupled-cluster types of wave functions) calculation of the oneparticle density matrix can be more difficult than repeated application of the energy-derivative method.

In this report we examine the utility of an approximate method for evaluation of properties based on second-order Hylleraas variational perturbation theory wave functions (and related wave functions) and QDVPT wave functions. The properties are formulated as the derivative of the second-order energy expression with respect to the perturbation strength. We neglect derivatives of the molecular orbital basis with respect to the perturbation. Due to the form of the energy expression used, a reduced density matrix can be defined. A similar method for property evaluation has been used for coupled-pair functional theory wave functions. ${ }^{15}$ Results are compared for the three systems examined previously in our tests of variational perturbation theory. ${ }^{18}$ Variational perturbation theory, QDVPT, multireference linearized coupled-cluster, ${ }^{5-7}$ and CI results are also presented for the model system examined in Secs. II and III which is designed to assess the effects of size inconsistency on the energy and first-order properties. 


\section{VARIATIONAL PERTURBATION THEORY AND SIZE INCONSISTENCY}

In this section we discuss the source of the size inconsistency in variational perturbation theory and introduce a simple model that illustrates this problem. We first review the relevant equations.

We adopt the following conventions for designating the configuration space. $\Psi_{0}$ designates the zeroth-order reference function for VPT or MRLCC. $\Psi_{0}$ need not be the lowest root of the zeroth-order space. When the reference space contains two functions, we designate the orthogonal complement of $\Psi_{0}$ as $\Psi_{c}$. When the entire reference space is being considered, we designate a member of this space as $\Psi_{0}^{i}$ ( $i=1, \ldots, n$ ), where $n$ is the number of functions in the reference space. Finally, when the reference space functions and the single and double excitations from the refrence space are being considered together, these functions are designated as $\Phi_{k} \quad(k=1, \ldots, N)$ with the first $n \Phi_{k}$ being the reference space functions. With these definitions the configuration space is partitioned into three sets:

$$
\begin{aligned}
& \Psi_{0}=\Psi_{0}^{1}=\Phi_{1}=\sum_{i=1}^{n} d_{0}^{i} \phi_{i}, \\
& \Psi_{0}^{k}=\Phi_{k}=\sum_{i=1}^{n} d_{k}^{i} \phi_{i}, \quad k=2, \ldots, n, \\
& \Phi_{k}=\phi_{k}, \quad k=n+1, \ldots, N .
\end{aligned}
$$

The $N-n \phi_{k}$ are all the single and double excitations relative to the reference configurations. The projection operators for these three spaces are denoted $P, Q_{0}$, and $Q_{1}$, respectively. The sum of $\mathbf{Q}_{0}$ and $\mathbf{Q}_{1}$ is denoted as $\mathbf{Q}$. The Hamiltonian is partitioned as

$$
\begin{aligned}
& H_{\mathrm{o}}=\mathbf{P} H \mathbf{P}+\mathbf{Q} H \mathbf{Q}, \\
& V=\mathbf{P} H \mathbf{Q}+\mathbf{Q} H \mathbf{P} .
\end{aligned}
$$

Using Eqs. (1) and (2) and assuming intermediate normalization $\left(\left\langle\Psi_{0} \mid \Psi\right\rangle=1\right)$, the expression for the second-order correction to the variational perturbation theory energy is obtained as ${ }^{10-18}$

$$
E_{2}=2\left\langle\Psi_{0}|H| \Psi_{1}\right\rangle+\left\langle\Psi_{1}\left|H-E_{0}\right| \Psi_{1}\right\rangle
$$

with $E_{0}=\left\langle\Psi_{0}|H| \Psi_{0}\right\rangle$ and $\Psi_{1}$ composed of the $\Phi_{k}$ $(k=2, \ldots, N)$. That is, $\Psi_{1}$ is defined as

$$
\Psi_{1}=\sum_{k=2}^{N} C_{k} \Phi_{k} .
$$

The variation of $E_{2}$ with respect to the $C_{k}(k=2, \ldots, N)$ yields

$$
0=\left\langle\Phi_{k}|H| \Psi_{0}\right\rangle+\left\langle\Phi_{k}\left|H-E_{0}\right| \Psi_{1}\right\rangle
$$

or, in matrix form,

$$
\mathbf{Q} H \mathbf{P}+\mathbf{Q}\left(H-E_{0}\right) \mathbf{Q C}=0 .
$$

Equations (3) and (6) are the defining equations for variational perturbation theory. We note that when Eq. (6) is satisfied the expression for $E_{2}$ reduces to

$$
E_{2}=\left\langle\Psi_{0}|H| \Psi_{1}\right\rangle
$$

which is identical to the usual Rayleigh-Schrödinger expression for the second-order correction to the energy.

The solution of Eq. (6) is the point at which the intruder state problems of the VPT equations can be seen formally. In general, Eq. (6) has a solution when the determinant of $\mathbf{Q}\left(H-E_{0}\right) \mathbf{Q}$ is nonzero. ${ }^{24}$ By performing a unitary transformation on $\mathbf{Q} H \mathbf{Q}$ to diagonalize it, one sees that this condition is equivalent to requiring that no eigenvalue of $\mathbf{Q} H \mathbf{Q}$ be equal to $E_{0}$. Near points where an eigenvalue of $\mathbf{Q} H \mathbf{Q}$ equals $E_{0}$ one expects the solutions of Eq. (6) to behave badly. The problem for VPT is that when the orthogonal complement of the reference space is included in $\mathbf{Q}$ one can always expect a crossing of an eigenvalue of $\mathbf{Q} H \mathbf{Q}$ and $E_{0}$ as the system size increases. This is because all single and double excitations relative to the reference space are included in $\mathbf{Q}$ and these single and double excitations correlate the orthogonal complement configurations as well as $\Psi_{0}$. As the correlation energy in the system increases, the lowest eigenvalue of $\mathbf{Q} H \mathbf{Q}$ (dominated by an orthogonal complement function for large systems ) can be made to sweep through $E_{0}$, causing instabilities in the resulting solutions. Thus the orthogonal complement functions (dressed by their interactions with the single and double excitations in $H_{0}$ ) become intruder states ${ }^{4}$ as the system size is increased for this nondegenerate treatment. Intruder state effects are less likely to occur for MRLCC since the orthogonal complement functions are excluded from $\mathbf{Q}$, and the single and double excitations do not correlate each other to the extent that they do $\mathbf{Q}_{0}$. Therefore, in MRLCC the lowest eigenvalue of $\mathbf{Q} H \mathbf{Q}$ can remain separated from $E_{0}$ even as the system size increases. To illustrate these features we introduce a simple model system.

The model system we wish to consider is the lowest ' $A_{\text {I }}$ state of $\mathrm{CH}_{2} \mathrm{He}_{n}$, where $\mathrm{CH}_{2}$ is at the equilibrium geometry for its lowest ' $A_{1}$ state and the He are well separated from one another and from $\mathrm{CH}_{2}$. For simplicity we assume two AOs are centered on each $\mathrm{He}$. Our treatment will be based on a two-configurational description of $\mathrm{CH}_{2} \mathrm{He}_{n}$, the two configurations differing only in the orbitals occupied by the $\mathrm{CH}_{2}$ lone pair electrons. The occupied and virtual orbitals are assumed localized on their respective centers; the SCF results reported below support this assumption. All doubly excited configurations on the He relative to both reference configurations and all double excitations on $\mathrm{CH}_{2}$ will be considered.

We designate the optimal two-configuration SCF function as $\Psi_{0}$ (with energy $E_{0}$ ) and its orthogonal complement in the reference space $\Psi_{c}$ (with energy $E_{c}$ ). Of the double excitations out of $\mathrm{He}$ orbitals, only those involving excitation of a pair of electrons on one $\mathrm{He}$ into an orbital on the same He will have nonzero matrix elements with $\Psi_{0}$, due to the assumed large distance between the He. Furthermore, for the two configurations corresponding to a double excitation on a given He one can transform to a new set of functions $\phi_{0}^{i}$ and $\phi_{c}^{i}$ (using the two-configuration SCF CI coefficients) that have the following properties:

$$
\begin{aligned}
& \left\langle\Psi_{0}|H| \Psi_{0}\right\rangle=E_{0}, \quad\left\langle\Psi_{c}|H| \Psi_{c}\right\rangle=E_{c}, \\
& \left\langle\Psi_{0}|H| \Psi_{c}\right\rangle=0 \quad\left\langle\Psi_{0}|H| \phi_{0}^{i}\right\rangle=H_{\mathrm{O}, \mathrm{He}}, \\
& \left\langle\Psi_{c}|H| \phi_{0}^{i}\right\rangle=0, \quad\left\langle\Psi_{0}|H| \phi_{c}^{i}\right\rangle=0, \\
& \left\langle\Psi_{c}|H| \phi_{c}^{i}\right\rangle=H_{c, \mathrm{He}}=H_{0, \mathrm{He}}, \\
& \left\langle\phi_{0}^{i}|H| \phi_{0}^{i}\right\rangle=E_{0}+\epsilon_{\mathrm{He}}, \quad\left\langle\phi_{c}^{i}|H| \phi_{0}^{i}\right\rangle=0 .
\end{aligned}
$$


In Eq. (8) $\epsilon_{\mathrm{He}}$ is the energy of the doubly excited configuration on He relative to the ground state SCF value. We also make use of $\epsilon_{c}=\left(E_{c}-E_{0}\right)$. Finally, we denote the set of doubly excited configurations on $\mathrm{CH}_{2}$ as (the vector) $\phi_{\mathrm{CH} 2}$, the associated vectors of connecting matrix elements being $\mathrm{H}_{0, \mathrm{CH} 2}$ and $\mathbf{H}_{c, \mathrm{CH} 2}$. Note that these double excitations do not connect with the He double excitations.

Since the $\mathrm{He}$ are equivalent it is clear that each $\mathrm{He}$ double excitation will enter $\Psi$ with the same coefficient, and that one can write $\Psi$ in the form

$$
\begin{aligned}
\Psi= & \left|\Psi_{0}\right\rangle+C_{c}\left|\Psi_{c}\right\rangle+C_{\mathrm{CH} 2}\left|\phi_{\mathrm{CH} 2}\right\rangle+C_{\mathrm{He}}^{0}\left|\chi_{0}\right\rangle \\
& +C_{\mathrm{He}}^{c}\left|\chi_{c}\right\rangle,
\end{aligned}
$$

where $C_{\mathrm{CH} 2}$ is a vector of coefficients and we have defined the normalized functions $\chi_{0}$ and $\chi_{c}$ as

$$
\begin{aligned}
& \chi_{0}=\sum_{i=1}^{n} \phi_{0}^{i} / \sqrt{ } n, \\
& \chi_{c}=\sum_{i=1}^{n} \phi_{c}^{i} / \sqrt{ } n .
\end{aligned}
$$

Note that $n$ indicates the number of $\mathrm{He}$. With these definitions the VPT equations defining the coefficients [Eq. (6)] become

$$
\begin{gathered}
\left(\begin{array}{cccc}
\epsilon_{\mathrm{He}} & 0 & 0 & 0 \\
0 & \epsilon_{c}+\epsilon_{\mathrm{He}} & V n H_{0, \mathrm{He}} & 0 \\
0 & V n H_{0, \mathrm{He}} & \epsilon_{c} & H_{c, \mathrm{CH} 2} \\
0 & 0 & H_{c, \mathrm{CH} 2} & H_{\mathrm{CH} 2}-E_{0}
\end{array}\right)\left(\begin{array}{c}
C_{\mathrm{He}}^{0} \\
C_{\mathrm{He}}^{c} \\
C_{c} \\
C_{\mathrm{CH} 2}
\end{array}\right) \\
=-\left(\begin{array}{c}
V n H_{0, \mathrm{He}} \\
0 \\
0 \\
H_{0, \mathrm{CH} 2}
\end{array}\right) .
\end{gathered}
$$

With the definitions

$$
\begin{aligned}
& H_{0,0}^{e}=H_{0, \mathrm{CH} 2}\left(E_{0}-H_{\mathrm{CH} 2}\right)^{-1} H_{\mathrm{CH} 2,0}, \\
& H_{c, c}^{e}=\epsilon_{c}+H_{c, \mathrm{CH} 2}\left(E_{0}-H_{\mathrm{CH} 2}\right)^{-1} H_{\mathrm{CH} 2, c}, \\
& H_{0, c}^{e}=H_{c, 0}^{e}=H_{c, \mathrm{CH} 2}\left(E_{0}-H_{\mathrm{CH} 2}\right)^{-1} H_{\mathrm{CH} 2,0},
\end{aligned}
$$

Eq. (11) can be solved to yield

$$
\begin{aligned}
C_{\mathrm{He}}^{0}= & -\sqrt{ } n H_{\mathrm{O}, \mathrm{He}} / \epsilon_{\mathrm{He}}, \\
C_{\mathrm{He}}^{c}= & \sqrt{ } n H_{0, \mathrm{He}} H_{c, 0}^{e} / D, \\
C_{c}=- & H_{\mathrm{O}, c}^{e}\left(\epsilon_{\mathrm{c}}+\epsilon_{\mathrm{He}}\right) / D, \\
C_{\mathrm{CH} 2}= & \left(E_{0}-H_{\mathrm{CH} 2}\right)^{-1} H_{\mathrm{CH} 2,0} \\
& \quad-\left(E_{0}-H_{\mathrm{CH} 2}\right)^{-1} H_{\mathrm{CH} 2 . c} H_{c, 0}^{e}\left(\epsilon_{c}+\epsilon_{\mathrm{He}}\right) / D
\end{aligned}
$$

with

$$
D=H_{c, c}^{e}\left(\epsilon_{c}-\epsilon_{\mathrm{He}}\right)-n H_{0, \mathrm{He}}^{2} .
$$

The total energy $E=E_{0}+\left\langle\Psi_{0}|H| \Psi_{1}\right\rangle$ becomes

$$
\begin{aligned}
E= & E_{0}-n\left|H_{0, \mathrm{He}}\right|^{2} / \epsilon_{\mathrm{He}}-H_{0, \mathrm{CH} 2}\left(H_{\mathrm{CH} 2}-E_{0}\right)^{-1} \\
& \times H_{\mathrm{CH} 2, \mathrm{O}}-\left|H_{0, c}^{e}\right|^{2}\left(\epsilon_{c}-\epsilon_{\mathrm{He}}\right) / D .
\end{aligned}
$$

The last term in Eq. (15) manifests the size inconsistency in variational perturbation theory for the present model system. The $D /\left(\epsilon_{c}+\epsilon_{\mathrm{He}}\right)$ factor in the last term of Eq. (15) approximately represents the energy of $\Psi_{c}$ after being corre- lated by the configurations in $\phi_{\mathrm{CH} 2}$ and $\chi_{\mathrm{c}}$. This quantity changes with increasing $\mathrm{He}$, while the factor multiplying it does not, thus leading to size inconsistency. In addition, this term also leads to the numerical instability in the VPT equations with increasing $\mathrm{He}$. For the present model, $H_{c, c}^{e}\left(\epsilon_{\mathrm{c}}+\epsilon_{\mathrm{He}}\right)$ is positive and since $-n H_{\mathrm{O}, \mathrm{He}}^{2}$ is negative, $D$ will go thrugh zero as $n$ is increased. At the point $D=0$, the VPT equations have no solution, and near this point they are unstable with respect to variation of $n$. Thus, as noted above, the dressed $\Psi_{c}$ becomes an intruder state in VPT. In the computations presented below it will be seen that this singularity is encountered between 9 and $13 \mathrm{He}$ for the basis sets used. However, it will be shown that this singularity does not have a large effect on the total energy, and that the largest errors actually occur in the one-electron properties.

For cases where $D$ is not near zero we can expand the denominator in Eq. (15) to obtain

$$
\begin{aligned}
E \simeq E_{0}-n\left|H_{0, \mathrm{He}}\right|^{2} / \epsilon_{\mathrm{He}}-H_{0, \mathrm{CH} 2}\left(H_{\mathrm{CH} 2}-E_{0}\right)^{-1} & \times H_{0, \mathrm{CH} 2}-\left|H_{c, 0}^{e}\right|^{2} / H_{c, c}^{e}-n\left|H_{0, \mathrm{He}}\right|^{2}\left|H_{c, 0}^{e}\right|^{2} / \\
& \left\{\left(H_{c, c}^{e}\right)^{2}\left(\epsilon_{c}-\epsilon_{\mathrm{He}}\right)\right\} .
\end{aligned}
$$

The second and third terms of Eq. (16) present the secondorder Rayleigh-Schrödinger contributions to the energy lowering based on the direct coupling of the $\mathrm{He}$ and $\mathrm{CH}_{2}$ double excitations to $\Psi_{0}$. The last two terms represent coupling of $\Psi_{c}$ to $\Psi_{0}$ through $\phi_{\mathrm{CH} 2}$. The direct coupling between $\Psi_{c}$ and $\Psi_{0}$ is zero when $\Psi_{0}$ is an eigenfunction of $H_{0}$. When the He are well separated from $\mathrm{CH}_{2}$ the coupling of $\Psi_{c}$ to $\Psi_{0}$ through the He double excitations is also zero. However, since one cannot transform away the coupling of $\Psi_{0}$ and $\Psi_{c}$ through the double excitations on $\mathrm{CH}_{2}$, this coupling will remain.

When no $\mathrm{CH}_{2}$ double excitations are included in the present model the effective coupling between $\Psi_{0}$ and $\Psi_{c}$ is zero and variational perturbation theory yields the same size-consistent result as MRLCC. However, in this model the He double excitations are least like "normal" correlating configurations in most molecular calculations. That is, in general, $\Psi_{0}$ and $\Psi_{c}$ will be coupled through most or all of the double excitations. Thus, for a multireference case, these effects are always present for variational perturbation theory, and intruder state problems can arise whenever the correlation energy is larger than the energy separation between $\Psi_{0}$ and an orthogonal complement function. This fact suggests that VPT should be modified for cases were the correlation energy is substantial.

\section{QUASIDEGENERATE VARIATIONAL PERTURBATION THEORY (QDVPT)}

Variational perturbation theory and most other multireference coupled-cluster or perturbation methods ${ }^{5,6,9-12,25}$ treat the zeroth-order space as a multiconfigurational nondegenerate space. Thus, the orthogonal complement functions are treated as minor contributors to the final wave function. However, there are occasions where such an approach may be inadequate. One example was given in Sec. II, where inclusion of $\Psi_{c}$ in $\Psi_{1}$ led to size-inconsistency effects because $\Psi_{c}$ is coupled to all single and double excitations. A 
second example occurs where correlation significantly alters the relative weights of the configurations comprising $\Psi_{0}$ in the final wave function. In this case neglect of the orthogonal complement configurations will lead to errors. Alternatively, inclusion of the orthogonal complement functions via VPT will lead to large (usually inaccurate) coefficients for these functions because VPT is only first order in its treatment of the corrections to $\Psi$.

In such cases it is important to be able to treat all the reference functions on a more equal basis, thus moving from a nondegenerate to a quasidegenerate formalism. Several groups have discussed ${ }^{3,7,8,13,26-28}$ and applied methods of this type ${ }^{8.13,14}$ Below we present a modification to variational perturbation theory based on an effective Hamiltonian constructed from a multireference zeroth-order space. It is derived using partitioning theory ${ }^{29}$ applied to the full $\mathrm{CI}$ Hamiltonian matrix and is aimed at treating the quasidegenerate problem. It also alleviates the problems encountered in nondegenerate multireference perturbation theory caused by the crossing of $E_{0}$ by eigenvalues of $H_{0}$ when the orthogonal complement of $\Psi_{0}$ is included in $\mathbf{Q}$. The method will be shown to reduce to VPT when the effective interaction between zeroth-order configurations is weak, and to MRLCC when the effective interaction is zero. But, since the current method treats the interaction between the reference functions to all orders it remains stable when strong zerothorder mixing occurs. It will be shown that the model is not strictly size consistent, due to an assumption which eliminates the dominant intruder state effects. Nevertheless, in applications of the model its size inconsistency will be shown to be much weaker than that of comparable CI calculations.

\section{A. Method}

The full CI Hamiltonian is partitioned into $\mathbf{P}, \mathbf{Q}$, and $\mathbf{R}$ spaces, in a similar way to that done for VPT. This partitioning was used by Kutzelnigg in work on perturbation theory. ${ }^{30}$ Based on this partitioning we obtain

$$
\begin{aligned}
& \mathbf{H}_{0}=\mathbf{P H P}+\mathbf{Q H Q}+\mathbf{R H R}, \\
& \mathbf{V}=\mathbf{P H Q}+\mathbf{Q H P}+\mathbf{Q H R}+\mathbf{R H Q}+\mathbf{P H R}+\mathbf{R H P} .
\end{aligned}
$$

If one assumes that $\mathbf{P}$ comprises all of the reference functions, $\mathbf{Q}$ all single and double excitations from $\mathbf{P}$, and $\mathbf{R}$ all higher excitations from $\mathbf{P}$, then the fifth and sixth terms of $\mathbf{V}$ in Eq. (17) are zero. The CI equations for this partitioning can be written as

$$
\left(\begin{array}{ccc}
\text { PHP } & \text { PHQ } & 0 \\
\text { QHP } & \text { QHQ } & \text { QHR } \\
0 & \text { RHQ } & \text { RHR }
\end{array}\right)\left(\begin{array}{l}
C_{P} \\
C_{Q} \\
C_{R}
\end{array}\right)=E\left(\begin{array}{l}
C_{P} \\
C_{Q} \\
C_{R}
\end{array}\right) .
$$

We rearrange Eq. (18) using

$$
C_{\mathbf{R}}=\{\mathbf{R}(\mathbf{E}-\mathbf{H}) \mathbf{R}\}^{-1} \mathbf{R H Q C}
$$

to yield

$$
\begin{gathered}
\left(\begin{array}{cc}
\text { PHP-E } & \text { PHQ } \\
\text { QHP } & \text { QHQ }+ \text { QHR }(R(E-H) R\}^{-1} R H Q-E
\end{array}\right) \\
\left(\begin{array}{l}
C_{\mathbf{P}} \\
C_{\mathbf{Q}}
\end{array}\right)=0
\end{gathered}
$$

where $\{\mathbf{R}(\mathbf{E}-\mathbf{H}) \mathbf{R}\}^{-1}$ is shorthand for the Lowdin $T$ matrix $^{29}$ defined as $\mathbf{R}[\boldsymbol{\alpha}(\mathbf{P}+\mathbf{Q})+\mathbf{R}(\mathbf{E}-\mathbf{H}) \mathbf{R}]^{-1} \mathbf{R}$, where $\boldsymbol{\alpha}$ is a constant. Note that $[\boldsymbol{\alpha}(\mathbf{P}+\mathbf{Q})+\mathbf{R}(\mathbf{E}-\mathbf{H}) \mathbf{R}]^{-\mathbf{1}}$ is block diagonal so that $\mathbf{P}[\boldsymbol{\alpha}(\mathbf{P}+\mathbf{Q})+\mathbf{R}(\mathbf{E}-\mathbf{H}) \mathbf{R}]^{-1} \mathbf{R}$ and $\mathbf{Q}[\boldsymbol{\alpha}(\mathbf{P}+\mathbf{Q})+\mathbf{R}(\mathbf{E}-$ $\mathbf{H}) \mathbf{R}]^{-1} \mathbf{R}$ equal zero. At this point, Eq. (20) is equivalent to the full $\mathrm{CI}$ equations and is impractical to solve, due to the coupling of the single and double excitations $(\mathbf{Q})$ to all higher excitations. A means of truncating the equations is needed. We do so in the following way. For the root within the reference space most like the state of interest we define $E_{0}=\left\langle\Psi_{0}|H| \Psi_{0}\right\rangle$ and set $E=E_{0}+E_{\text {corr }}$ in the block of $H$ over the $\mathbf{Q}$ configurations. (Various choices for $\Psi_{0}$ will be discussed below.) Realizing that the effect of higher excitations contained in the term $\mathbf{Q H R}(\mathbf{R}(\mathbf{E}-\mathbf{H}) \mathbf{R}\}^{-1} \mathbf{R H Q}$ is to dress (or correlate) the single and double excitations, we approximate the $\mathbf{Q}$ block by replacing this term by the diagonal matrix $\mathbf{E}_{\text {corr }}$. That is, we assume that the correlation energy for the single and double excitations is similar to that of the state of interest. [This is not completely accurate, since the $E$ in the term QHR $(\mathbf{R}(\mathbf{E}-\mathbf{H}) \mathbf{R}\}^{-1} \mathbf{R H Q}$ is that of the state under consideration, and thus this term is not entirely equivalent to the correlation energy of each of the single and double excitations.] With this approximation Eq. (20) becomes

$$
\left(\begin{array}{cc}
\text { PHP-E } & \text { PHQ } \\
\text { QHP } & \text { QHQ-E }
\end{array}\right)\left(\begin{array}{l}
C_{P} \\
C_{Q}
\end{array}\right)=0 .
$$

Equation (21) can itself be rearranged using

$$
\mathbf{C}_{\mathbf{Q}}=\left\{\mathbf{Q}\left(\mathbf{E}_{0}-\mathbf{H}\right) \mathbf{Q}\right\}^{-1} \mathbf{Q H P C} \mathrm{C}_{\mathrm{P}}
$$

[where $\left\{\mathbf{Q}\left(\mathbf{E}_{0}-\mathbf{H}\right) \mathbf{Q}\right]^{-1}$ is defined analogously to $\left.\{\mathbf{R}(\mathbf{E}-\mathbf{H}) \mathbf{R}\}^{-1}\right]$ to give the effective Schrödinger equation

$$
\left.\left\{\mathbf{P H P}+\mathbf{P H Q}\left(\mathbf{Q}\left(\mathbf{E}_{\mathbf{0}}-\mathrm{H}\right) \mathbf{Q}\right\}^{-1} \mathbf{Q H P}\right\} \mathrm{C}_{\mathbf{P}}\right\}=\mathrm{EC}_{\mathbf{P}} .
$$

The effective Hamiltonian defined by Eq. (23) is Hermitian and is defined only over the reference space. We stress that, as in VPT or MRLCC, QHQ is the full interaction matrix for the single and double excitations, not just the diagonal elements as in the $B_{K}{ }^{31}$ or Rayleigh-Schrödinger $B_{K}{ }^{32}$ approaches. The direct method of solution for QDVPT uses this partitioning and solves Eqs. (22) and (23). In contrast to MRLCC where a specific $C_{P}$ is chosen and Eq. (22) is solved, $C_{P}$ is not known in QDVPT. As a result one must essentially solve $n$ MRLCC problems, one for each function in the $\mathbf{P}$ space. We have implemented the solution of the QDVPT equations using this direct method and have obtained QDVPT wave functions by diagonalizing Eq. (23). While this approach is possible, it turns out to be unwieldy for large reference spaces, requiring essentially $n$ times the work of a VPT or MRLCC calculation. In the Appendix we present an iterative method for solving Eq. (21) which proves to be much more efficient. This treatment takes only somewhat more effort than a MRLCC or VPT calculation.

Since we require $E_{0}$ for the root of the reference space most like the state of interest, we normally begin the calculation by diagonalizing $H_{0}$ over the reference space and defining $H$ in terms of the eigenfunctions of $H_{0}$ in the reference space. (This is not a necessary step, since given an $E_{0}$ the 
QDVPT equations are invariant to a unitary transformation within the reference space. Rather, we perform this operation to make obvious the relation of QDVPT to MRLCC and VPT, where this prediagonalization of the reference space is necessary.) When this is done, PHP is diagonal and any coupling that occurs between the zeroth-order states now arises due to the $\mathbf{P H Q}\left(\mathbf{Q}\left(\mathbf{E}_{\mathbf{0}}-\mathbf{H}\right) \mathbf{Q}\right\}^{-1} \mathbf{Q H P}$ term. If this term becomes large, significant mixing will occur within the $\mathbf{P}$ space. In this case, $E_{0}$ is no longer the appropriate reference energy to use in solving Eq. (21) and it is best to iterate these equations, using the new $E_{0}$ defined by $E_{0}=\mathbf{C}_{\mathbf{P}}^{\mathbf{t}} \mathbf{H}_{\mathbf{0}} \mathbf{C}_{\mathbf{P}}$ with $\mathbf{C}_{\mathbf{P}}^{\mathbf{t}} \mathbf{C}_{\mathbf{P}}=1$ (note this expression contains $H_{0}$ and not the effective $H$ ), repeating this process until convergence is reached.

In the other extreme, when both PHP and PHQ $\left\{Q\left(E_{0}-\mathbf{H}\right) \mathbf{Q}\right\}^{-1} \mathbf{Q H P}$ yield no coupling within the reference space, Eqs. (22) and (23) can be shown to yield the MRLCC energy and wave function for the root corresponding to the $E_{0}$ chosen. The diagonal elements corresponding to the remaining configurations are

$$
H_{j j}=E_{0}^{j}+\left\langle\Psi_{0}^{j}\left|\mathbf{H Q}\left\{\mathbf{Q}\left(\mathbf{E}_{0}-\mathbf{H}\right) \mathbf{Q}\right\}^{-1} \mathbf{Q H}\right| \Psi_{0}^{j}\right\rangle .
$$

Thus the remaining diagonal elements do not correspond to the MRLCC energies for these roots, due to the presence of $E_{0}$ in their effective matrix elements, rather than $E_{0}^{j}$. However, it is just this approximation that inhibits the problems encountered with intruder states. Were the effective Hamiltonian to reduce to separate MRLCC calculations on each root when no effective coupling existed, one would have quite high values of $E_{0}^{j}$ in the effective matrix elements, leading to crossings with eigenvalues of QHQ and instabilities in the equations as were encountered in VPT.

\section{B. Relation to other methods}

The present method is somewhat similar to the $B_{K}{ }^{31}$ and Rayleigh-Schrödinger $B_{K}{ }^{32}$ methods. Referring to Eq. (20), the first difference between QDVPT and the $B_{K}$ method is that in QDVPT we substitute $-E_{0}$ for $\{-E$ + QHR $\left.\{\mathbf{R}(\mathbf{E}-\mathbf{H}) \mathbf{R}\}^{-1} \mathbf{R H Q}\right\}$, whereas the $B_{K}$ method neglects $\mathbf{Q H R}(\mathbf{R}(\mathbf{E}-\mathbf{H}) \mathbf{R}\}^{-1} \mathbf{R H Q}$ and retains $E$, thus yielding a size inconsistency similar to that of MRSDCI The second difference between $B_{K}$ and QDVPT is that the $B_{K}$ method only retains the diagonal elements in the QHQ block, whereas QDVPT retains the full QHQ matrix. Rayleigh-Schrödinger $B_{K}$ perturbation theory differs from QDVPT only in the second of these two ways, since it makes the same substitution of $-E_{0}$ for $\{-E+\mathbf{Q H R}$ $\left.\{\mathbf{R}(\mathbf{E}-\mathbf{H}) \mathbf{R}\}^{-1} \mathbf{R H Q}\right\}$ as QDVPT does. The expected improvement in accuracy of QDVPT over Rayleigh-Schrödinger $B_{K}$ perturbation theory should be similar to that found in proceeding from conventional Rayleigh-Schrödinger PT to the nondegenerate second-order VPT defined in Sec. II and Ref. 18.

The relation between the present method and MRLCC was discussed above. The difference between QDVPT and MRSDCI consists in that CI neglects $\mathbf{Q H R}(\mathbf{R}(\mathbf{E}-\mathbf{H}) \mathbf{R}]^{-1} \mathbf{R H Q}$ in the $\mathbf{Q}$ block, but retains $E$, rather than substituting $E_{0}$. Variational perturbation theory will be shown below to be related to QDVPT in a perturbative sense; i.e., by solving the effective Schrödinger equation perturbatively (when such an expansion is appropriate) one recovers the VPT results in low orders.

Finally, QDVPT can be shown to be similar to the recently introduced multireference averaged coupled-pair functional method of Gdanitz and Ahlrichs. ${ }^{15}$ In the multireference averaged coupled-pair functional method the configuration space (outside $\Psi_{0}$ ) is broken up into two parts: $\Psi_{a}$ comprises all configurations with orbital occupations that are the same as the reference configurations outside the active space, and $\Psi_{e}$ is made up of all other configurations. A modified CISD energy expression is used in which the normalization terms in the denominator corresponding to $\Psi_{a}$ and $\Psi_{c}$ are weighted by the factors $g_{a}$ and $g_{c}$, respectively. Gdanitz and Ahlrichs choose $g_{a}=1$ (reasoning that no renormalization is needed for the a space) and $g_{e}=2 / n$, where $n$ is the number of electrons being correlated. The definition of $g_{e}$ is obtained by requiring that the energy functional employed have the proper dependence on particle number for a system of separated electron pairs. QDVPT can be obtained from their energy functional by setting $g_{c}=0$ and $g_{a}=1$.

\section{Application of QDVPT to $1^{\mathbf{1}} \boldsymbol{A}_{1} \mathrm{CH}_{\mathbf{2}}(\mathrm{He})_{n}$}

Using the same definitions of the components of $\Psi$ as were used in Sec. II we now have

$$
\begin{aligned}
& \mathbf{P}=\left|\Psi_{0}\right\rangle\left\langle\Psi_{0}|+| \Psi_{c}\right\rangle\left\langle\Psi_{c}\right|, \\
& \mathbf{Q}=\left|\phi_{\mathrm{CH} 2}\right\rangle\left\langle\phi_{\mathrm{CH} 2}|+| \chi_{0}\right\rangle\left\langle\chi_{0}|+| \chi_{c}\right\rangle\left\langle\chi_{c}\right|
\end{aligned}
$$

With the definitions for the Hamiltonian matrix elements used in Sec. II we obtain the effective Hamiltonian

$$
\begin{aligned}
\mathbf{H}_{\mathrm{eff}} & -\mathbf{E}_{\mathbf{0}} \\
& =\left(\begin{array}{cc}
H_{0,0}^{e}-n H_{0,10}^{2} / \epsilon_{\mathrm{He}} & H_{0, c}^{e} \\
H_{c, 0}^{e} & H_{c, c}^{e}-n H_{c, \gamma \mathrm{c}}^{2} /\left(\epsilon_{\mathrm{c}}+\epsilon_{\mathrm{He}}\right)
\end{array}\right) .
\end{aligned}
$$

Because the $\mathrm{He}$ do not interact with $\mathrm{CH}_{2}, H_{c, \gamma c}$ $=H_{0,10}=H_{0, H_{\mathrm{e}}}$. Defining the matrix elements of Eq. (26) as $M_{i j}$ and solving for the lowest root of the effective Schrödinger equation we obtain

$$
\begin{aligned}
E_{1}= & E_{0}+\left(M_{11}+M_{22}\right) / 2+\left[\left(M_{11}-M_{22}\right) / 2\right] \\
& \times\left[\left(1+4\left(M_{12}\right)^{2} /\left(M_{11}-M_{22}\right)\right]^{1 / 2}\right. \\
= & E_{0}+M_{11}+\left(M_{12}\right)^{2} /\left(M_{11}-M_{22}\right) \\
& -\left(M_{12}\right)^{4} /\left(M_{11}-M_{22}\right)^{3}+O\left[\left(M_{12}\right)^{6}\right]
\end{aligned}
$$

Substituting for the $M_{i j}$ one obtains

$$
\begin{aligned}
E_{1}= & E_{0}-n H_{0, \mathrm{He}}^{2} / \epsilon_{\mathrm{He}}-H_{0, \mathrm{CH} 2}\left(E_{0}-H_{\mathrm{CH} 2}\right)^{-1} H_{\mathrm{CH} 2,0} \\
& +\left|H_{c, 0}^{e}\right|^{2} /\left[H_{0,0}^{e}-H_{c, c}^{e}-n H_{0, \chi 0}^{2} /\right. \\
& \left.\epsilon_{\mathrm{He}}+n H_{c, \chi c}^{2} /\left(\epsilon_{c}+\epsilon_{\mathrm{He}}\right)\right] \\
& -\left|H_{c, 0}^{e}\right|^{4} /\left[H_{0,0}^{e}-H_{c, c}^{e}-n H_{0, \chi 0}^{2} / \epsilon_{\mathrm{He}}\right. \\
& +n H_{c, \chi c}^{2} /\left(\epsilon_{\mathrm{c}}+\epsilon_{\mathrm{He}}\right]^{3}+O\left(H_{i j}^{12}\right)
\end{aligned}
$$


For comparison with the VPT results we expand out the $M_{11}-M_{22}$ denominators around $H_{c, c}^{e}$ and obtain

$$
\begin{aligned}
E \simeq & E_{0}-n\left|H_{0, \mathrm{He}}\right|^{2} / \epsilon_{\mathrm{He}}-H_{0, \mathrm{CH} 2}\left(E_{0}-H_{\mathrm{CH} 2}\right)^{-1} H_{\mathrm{CH} 2,0} \\
& -\left|H_{c, 0}^{e}\right|^{2} / H_{c, c}^{e}+H_{0,0}^{e}\left|H_{c, 0}^{e}\right|^{2} /\left(H_{c, c}^{e}\right)^{2} \\
& -n\left|H_{0, \mathrm{He}}\right|^{2}\left|H_{c, 0}^{c}\right|^{2}\left\{1 /\left(\epsilon_{c}+\epsilon_{\mathrm{He}}\right)-1 / \epsilon_{\mathrm{He}}\right\} /\left(H_{c, c}^{e}\right)^{2} .
\end{aligned}
$$

The only differences between Eq. (28) and the VPT result are the fifth and seventh terms of Eq. (28), which do not appear in Eq. (16). Thus, in the regions where $1 / D$ or $1 /$ $\left(M_{11}-M_{22}\right)$ can be expanded as above, the VPT and QDVPT results are expected to be quite close.

A more natural choice about which to expand the QDVPT energy is $1 /\left(H_{c, c}^{e}-H_{0,0}^{e}\right)$, in which both $\Psi_{0}$ and $\Psi_{c}$ are dressed by their interactions with the $\mathrm{CH}_{2}$ double excitations. The fifth term in Eq. (28) arises because we instead expanded about $1 / H_{c, c}^{e}$, and the ratio $H_{0,0}^{e} / H_{c, c}^{e}$ gives information regarding the appropriateness of VPT for a given problem. When this factor becomes large one expects intruder state effects to be important. Note that QDVPT is still valid, it merely indicates that this choice for expansion of the QDVPT denominator is inappropriate. In fact, one need not perform any expansion in the QDVPT case and then the QDVPT energy expression is valid even when $\left(M_{11}-M_{22}\right)$ is zero. (In the calculations presented below we, of course, do not expand out the QDVPT energy expression. This expansion is performed here for illustrative purposes.) It should be noted that it is the presence of the factor $1 /\left(M_{11}-M_{22}\right)$ rather than $1 / D$ which contributes to the stability of the QDVPT equations. As shown above $D$ will go through zero as the number of He is increased, whereas one expects $\left(M_{11}-M_{22}\right)$ to be nearly constant with increasing He. When $D$ becomes small the VPT equations cannot be solved either perturbatively or exactly.

Finally, we comment on the lack of size consistency in QDVPT. Within the present simple model it can be shown that $M_{12}\left(=M_{21}\right)$ is constant as the number of $\mathrm{He}$ is increased. However, the difference between $M_{11}$ and $M_{22}$ will change as the number of $\mathrm{He}$ is increased. This is due to the presence of $E_{0}$ in the expression for $H_{c c}^{c}$, rather than $E_{c}$ as would be found in a MRLCC calculation on $\Psi_{c}$. That is, given that the $\mathrm{He}$ are well separated from $\mathrm{CH}_{2}$ one would expect the same He double excitation coefficients (and thus the same contribution to the correlation energy of each state) from the expressions $\left\{\mathbf{Q}\left(\mathbf{E}_{\mathbf{0}}-\mathbf{H}\right) \mathbf{Q}\right\}^{-1} \mathbf{Q H} \Psi_{0}$ and $\left\{\mathbf{Q}\left(\mathbf{E}_{\mathrm{c}}-\mathbf{H}\right) \mathbf{Q}\right\}^{-1} \mathbf{Q H} \Psi_{c}$. This is not the case for $\left\{\mathbf{Q}\left(\mathbf{E}_{0}-\mathbf{H}\right) \mathbf{Q}\right\}^{-1} \mathbf{Q H} \Psi_{\text {c }}$ which is the expression used in QDVPT to generate the effective matrix elements for the orthogonal complement to $\Psi_{0}$. This leads to the energy contribution per He to $M_{22}$ being somewhat different from that to $M_{11}$. In the calculations performed below $M_{11}-M_{22}$ becomes more negative with increasing $\mathrm{He}$, and in the limit of an infinite number of $\mathrm{He}$ in the present system the QDVPT result would reduce to an MRLCC calculation on the lowest state. However, it will also be shown below that this effect is quite small relative to the size inconsistency of truncated $\mathrm{CI}$ and should not be a major factor, even for calculations on quite large molecules.

\section{PROPERTY EVALUATION}

While the methods for evaluation of first-order oneelectron properties for VPT and QDVPT are formally similar, some differences exist between the two. We first present the theory for VPT and MRLCC and then proceed to QDVPT.

\section{A. VPT/MRLCC properties}

The full CI Hamiltonian for a fixed many-electron basis in the presence of the perturbation $G$ is written as

$$
\mathbf{H}=\mathbf{H}^{\mathrm{f}}+\mu G,
$$

where the superscript $f$ denotes the field-free Hamiltonian for the system of interest. We partition the field-dependent Hamiltonian in the usual way for VPT or MRLCC and $E_{0}$, $E_{2}, \Psi_{0}$, and $\Psi_{1}$ are then functions of the strength of the perturbation $\mu$. To the extent that $E_{0}+E_{2}$ for VPT or MRLCC is able to approximate the eigenvalue of the full CI matrix, the property $\langle G\rangle$ is given by $\partial E /\left.\partial \mu\right|_{\mu=0}$. The derivative of the total VPT or MRLCC energy with respect to $\mu$ evaluated at $\mu=0$ is

$$
\begin{gathered}
\frac{\partial E}{\partial \mu}=\frac{\partial E_{0}}{\partial \mu}+\frac{\partial E_{2}}{\partial \mu}, \\
\frac{\partial E_{2}}{\partial \mu}=\left\langle\frac{\partial \Psi_{0}}{\partial \mu}\left|H^{f}\right| \Psi_{1}\right\rangle+\left\langle\Psi_{0}|G| \Psi_{1}\right\rangle+\left\langle\Psi_{0}\left|H^{f}\right| \frac{\partial \Psi_{1}}{\partial \mu}\right\rangle .
\end{gathered}
$$

After some manipulations, one obtains the expression (for real wave functions)

$$
\begin{aligned}
\frac{\partial E}{\partial \mu}= & \left(1-\left|\Psi_{1}\right|^{2}\right\rangle\left\langle\Psi_{0}|G| \Psi_{0}\right\rangle+2\left\langle\Psi_{1}|G| \Psi_{0}\right\rangle \\
& +\left\langle\Psi_{1}|G| \Psi_{1}\right\rangle+2\left\langle\frac{\partial \Psi_{0}}{\partial \mu}\left|H^{f}-E_{0}-E_{2}\right| \Psi_{0}+\Psi_{1}\right\rangle \\
& -2\left|\Psi_{1}\right|^{2}\left\langle\Psi_{0}\left|H^{f}\right| \frac{\partial \Psi_{0}}{\partial \mu}\right\rangle .
\end{aligned}
$$

When $\Psi_{0}$ is an eigenfunction of $H_{0}^{f}$, the last term in Eq. (32) is zero. Furthermore, when $\Psi_{0}$ is an eigenfunction of $H_{0}^{f}$, $\partial \Psi_{0} / \partial \mu$ is easily obtained as $\left\{\mathbf{Q}_{0}\left(\mathbf{E}_{\mathbf{0}}^{\mathbf{f}}-\mathbf{H}^{\mathrm{f}}\right) \mathbf{Q}_{0}\right\}^{-1} \mathbf{G} \Psi_{0}$ and Eq. 32) becomes for VPT:

$$
\begin{aligned}
\frac{\partial E_{\mathrm{VPT}}}{\partial \mu}= & \left.\left.\left(1-\left|\Psi_{1}\right|^{2}\right)\right\rangle \Psi_{0}|G| \Psi_{0}\right\rangle \\
& +2\left\langle\Psi_{1}|G| \Psi_{0}\right\rangle+\left\langle\Psi_{1}|G| \Psi_{1}\right\rangle \\
& \left.-2 E_{2}\left\langle\Psi_{0}|G|\left\{\mathbf{Q}_{0}-\mathbf{E}_{0}^{\mathrm{f}}-\mathbf{H}^{\mathrm{f}}\right) \mathbf{Q}_{0}\right\}^{-1} \Psi_{1}\right\rangle,
\end{aligned}
$$

whereas for MRLCC Eq. (32) becomes

$$
\begin{aligned}
\frac{\partial E_{\mathrm{MRLCC}}}{\partial \mu}= & \left.\left.\left(1-\left|\Psi_{1}\right|^{2}\right)\right\rangle \Psi_{0}|G| \Psi_{0}\right\rangle \\
& +2\left\langle\Psi_{1}|G| \Psi_{0}\right\rangle+\left\langle\Psi_{1}|G| \Psi_{1}\right\rangle \\
& \left.+2\left\langle\Psi_{0}|G|\left\{\mathbf{Q}_{0}-\mathbf{E}_{0}^{\mathrm{f}}-\mathbf{H}^{\mathrm{f}}\right) \mathbf{Q}_{0}\right\}^{-1} \mathrm{H} \Psi_{1}\right\rangle .
\end{aligned}
$$

Alternatively, one could choose to not allow the coefficients defining $\Psi_{0}$ to change with application of the field. In this case $\partial \mathbf{P} / \partial \mu=\partial \mathbf{Q} / \partial \mu=0$ and the expression for both the VPT and MRLCC properties reduces to 


$$
\begin{aligned}
\frac{\partial E}{\partial \mu}= & \left.\left.\left(1-\left|\Psi_{1}\right|^{2}\right)\right\rangle \Psi_{0}|G| \Psi_{0}\right\rangle \\
& +2\left\langle\Psi_{1}|G| \Psi_{0}\right\rangle+\left\langle\Psi_{1}|G| \Psi_{1}\right\rangle
\end{aligned}
$$

We denote properties calculated using Eq. (35) as $\langle G\rangle_{\text {fix }}$ below, and refer to these fixed or frozen $\Psi_{0}$ properties, since the coefficients defining $\Psi_{0}$ are fixed at their zero-field values. The properties obtained from Eq. (35) are equivalent to those obtained using the modified expectation value

$$
\langle G\rangle=\left\langle\Psi_{0}|G| \Psi_{0}\right\rangle+\left\langle\Psi_{0}+\Psi_{1}\left|G-G_{0}\right| \Psi_{0}+\Psi_{1}\right\rangle,
$$

where $G_{0}=\left\langle\Psi_{0}|G| \Psi_{0}\right\rangle$. Equation (36) can be obtained in a somewhat different manner by writing

$$
\langle G\rangle=\left\langle\Psi_{0}+\Psi_{1}|G| \Psi_{0}+\Psi_{1}\right\rangle /\left\langle\Psi_{0}+\Psi_{1} \mid \Psi_{0}+\Psi_{1}\right\rangle
$$

expanding the denominator, and keeping terms up to second order in $\Psi_{t}$. The equivalence of Eqs. (35) and (36) when the coefficients defining $\Psi_{0}$ are fixed at their zero-field values arises because the $C_{j}$ are obtained variationally via $\mathrm{Eq}$. (6). This may be viewed as a modified Hellmann-Feynman theorem for the energy expression used above [Eq. (3)], with the assumption that the atomic and molecular orbitals are fixed.

In general, Eqs. (33) and (34) are expected to be more accurate than Eq. (35), since they take into account the variation of $\Psi_{0}$ with the perturbation strength. However, when $\Psi_{0}$ is not an eigenfunction of $H_{0}^{f}$ we have chosen to use Eq. (35) to evaluate molecular properties, thus using the form equivalent to the expectation value in $\mathrm{Eq}$. (36). We choose this due to the ambiguity in the derivative of the $d_{0}^{i}$ associated with an arbitrarily chosen $\Psi_{0}$. One could use standard first-order Rayleigh-Schrödinger perturbation theory to define $\partial \mathbf{d}_{0} / \partial \mu$, but we have chosen to discard the term arising from $\partial \mathbf{d}_{0} / \partial \mu$. In using Eqs. (32) or (34) an effective one-particle density matrix can be defined for the variational perturbation theory or MRLCC wave functions as

$\rho=\left(1-\left\langle\Psi_{1} \mid \Psi_{1}\right\rangle\right) \rho_{0}+\left(\rho_{01}+\rho_{10}\right)+\rho_{1}+\left(\rho_{01}^{X}+\rho_{10}^{X}\right)$

with $X$ either VPT or MRLCC and where

$$
\begin{aligned}
& \rho_{0}=\int \cdots \int d r_{2} \cdots d r_{M} \Psi_{0}^{*}\left(r_{1}, \ldots, r_{M}\right) \Psi_{0}\left(r_{1}, \ldots, r_{M}\right) \\
& \rho_{01}=\int \cdots \int d r_{2} \cdots d r_{M} \Psi_{0}^{*}\left(r_{1}, \ldots, r_{M}\right) \Psi_{1}\left(r_{1}, \ldots, r_{M}\right) \\
& \rho_{10}=\int \cdots \int d r_{2} \cdots d r_{M} \Psi_{1}^{*}\left(r_{1}, \ldots, r_{M}\right) \Psi_{0}\left(r_{1}, \ldots, r_{M}\right), \\
& \rho_{1}=\int \cdots \int d r_{2} \cdots d r_{M} \Psi_{1}^{*}\left(r_{1}, \ldots, r_{M}\right) \Psi_{1}\left(r_{1}, \ldots, r_{M}\right)
\end{aligned}
$$

and

$$
\begin{aligned}
\rho_{01}^{\mathrm{VPT}}= & -E_{2} \int \cdots \int d r_{2} \cdots d r_{M} \Psi_{0}^{*}\left(r_{1}, \ldots, r_{M}\right) \\
& \times\left\{\mathbf{Q}_{0}\left(\mathbf{E}_{\mathbf{0}}^{\mathrm{f}}-\mathbf{H}^{\mathrm{f}}\right) \mathbf{Q}_{\mathbf{0}}\right\}^{-\mathbf{1}} \Psi_{1}\left(r_{1}, \ldots, r_{M}\right),
\end{aligned}
$$

$$
\begin{aligned}
\rho_{10}^{\mathrm{VPT}}= & -E_{2} \int \cdots \int d r_{2} \cdots d r_{M}\left\{\mathbf{Q}_{0}\left(\mathbf{E}_{\mathbf{0}}^{\mathbf{f}}-\mathbf{H}^{\mathbf{f}}\right) \mathbf{Q}_{0}\right\}^{-\mathbf{1}} \\
\times & \Psi_{1}^{*}\left(r_{1}, \ldots, r_{M}\right) \Psi_{0}\left(r_{1}, \ldots, r_{M}\right) \\
\rho_{01}^{\mathrm{MRLCC}}= & \int \cdots \int d r_{2} \cdots d r_{M} \Psi_{0}^{*}\left(r_{1}, \ldots, r_{M}\right) \\
& \times\left(\mathbf{Q}_{0}\left(\mathbf{E}_{\mathbf{0}}^{\mathrm{f}}-\mathbf{H}^{\mathrm{f}}\right) \mathbf{Q}_{\mathbf{0}}\right\}^{-\mathbf{1}} \mathbf{H} \Psi_{1}\left(r_{1}, \ldots, r_{M}\right),(41 \mathrm{a}) \\
\rho_{10}^{\mathrm{MRLCC}}= & \int \cdots \int d r_{2} \cdots d r_{M}\left\{\mathbf{Q}_{0}\left(\mathbf{E}_{\mathbf{0}}^{\mathbf{f}}-\mathbf{H}^{\mathbf{f}}\right) \mathbf{Q}_{0}\right\}^{-\mathbf{1}} \mathbf{H} \\
& \times \Psi_{1}^{*}\left(r_{1}, \ldots, r_{M}\right) \Psi_{0}\left(r_{1}, \ldots, r_{M}\right) .
\end{aligned}
$$

When fixed properties are calculated $\rho$ is obtained using Eq. (42), with the various terms defined as in Eq. (39):

$$
\rho=\left(1-\left\langle\Psi_{1} \mid \Psi_{1}\right\rangle\right) \rho_{0}+\left(\rho_{01}+\rho_{10}\right)+\rho_{1} .
$$

One-electron properties are obtained via Eq. (43):

$$
\langle G\rangle=\operatorname{Tr}(\rho G) \text {. }
$$

Finally, we point out that there is an effective inconsistency in Eqs. (33) and (34) in the order of the terms included from the field-free wave function. Since $\Psi_{1}$ is the firstorder correction to the wave function, the expressions for $\langle G\rangle$ contain zeroth-order terms $\left(G_{0}\right)$, first-order terms $\left(\left\langle\Psi_{0}|G| \Psi_{1}\right\rangle\right)$, and some of the second-order terms $\left(\left\langle\Psi_{1}\left|G-G_{0}\right| \Psi_{1}\right\rangle\right)$. The other second-order terms, of the form $\left\langle\Psi_{0}|G| \Psi_{2}\right\rangle$, do not appear since the expression for the properties presented above is based on the second-order energy expression. These terms do not enter into the energy at second order.

\section{B. Quasidegenerate variational perturbation theory properties}

As was the case for VPT properties we obtain the QDVPT properties as derivatives of the total energy with respect to the strength of the applied perturbation. Beginning with the effective Schrödinger equation [Eq. (23)] we have

$$
E(\mu) C_{P}(\mu)=H_{\mathrm{eff}}(\mu) C_{P}(\mu),
$$

where the dependence on $\mu$ has been made explicit. Assuming that $C_{P}$ is normalized we have

$$
E(\mu)=C_{P}^{\prime}(\mu) H_{\text {eff }}(\mu) C_{P}(\mu) .
$$

From here on we drop the explicit reference to the $\mu$ dependence. We assume that the basis states for $H_{\text {eff }}$ are an arbitrary set of $\mu$-independent linear combinations of the reference space configurations. The case where $H_{\mathrm{eff}}$ and $C_{P}$ are defined in terms of field-dependent zeroth-order functions can be shown to be equivalent to what follows since, as long as the reference space configurations are fixed the field-dependent and field-independent zeroth-order states are related by a unitary transformation. Thus the effective Hamiltonians and $C_{P}$ in the two bases are related in the usual way and the energy is invariant to this transformation.

Traking the derivative of $E$ with respect to $\mu$ and using the facts that $C_{P}$ is an eigenfunction of $H_{\text {eff }}$ and that $\partial\left(C_{P}{ }_{P} C_{P}\right) / \partial \mu=0$ one obtains

$$
\langle G\rangle=\frac{\partial E}{\partial \mu}=C_{P}^{t} \frac{\partial H_{\mathrm{eff}}}{\partial \mu} C_{P} .
$$


In an entirely analogous manner to the procedure for VPT one obtains the effective one-electron density matrix (noting that $\partial \mathbf{Q} / \partial \mu=0$, since $\mathbf{P}$ contains $\Psi_{0}$ and its orthogonal complement)

$$
\rho=\rho_{0}+\left(\rho_{01}+\rho_{10}\right)+\rho_{1}-\left\langle\Psi_{1} \mid \Psi_{1}\right\rangle \rho_{0}^{0}
$$

when $E_{0}$ is defined by the eigenvector of $H_{0}$ in the reference space, or

$$
\begin{aligned}
\rho= & \rho_{0}+\left(\rho_{01}+\rho_{10}\right)+\rho_{1}-\left\langle\Psi_{1}|\Psi|\right\rangle \\
& \times\left\{\rho_{0}+2\left\langle C_{P}\left|H^{f}\right| \frac{\partial C_{P}}{\partial \mu}\right)\right\}
\end{aligned}
$$

when $E_{0}$ is defined self-consistently in terms of the final $C_{P}$. In either case $\rho_{0}, \rho_{01}, \rho_{10}$, and $\rho_{1}$ are given by Eq. (39) and

$$
\rho_{0}^{0}=\int \cdots \int d r_{2} \cdots d r_{M} \Psi_{0}^{0^{*}}\left(r_{1}, \ldots, r_{M}\right) \Psi_{0}^{0}\left(r_{1}, \ldots, r_{M}\right),
$$

where $\Psi_{0}^{0}$ is the eigenfunction of $H_{0}$ corresponding to $E_{0}$.

Note that due to the normalization chosen $\left(C_{P}^{t} C_{P}=1\right)$ the trace of $\rho_{0}$ is equal to the total electron density, as is the case for $\rho_{0}^{0}$ and $\rho$. With this choice of normalization we can write Eq. (47) (defining $\Psi_{\text {EVPT }}=\Psi_{0}+\Psi_{1}$ )

$$
\rho=\rho_{\mathrm{EVPT}}-\left\langle\Psi_{1} \mid \Psi_{1}\right\rangle \rho_{0}^{0}
$$

with

$$
\begin{aligned}
\rho_{\mathrm{EVPT}}= & \int \cdots \int d r_{2} \cdots d r_{M} \\
& \times \Psi_{\mathrm{EVPT}}^{*}\left(r_{1}, \ldots, r_{M}\right) \Psi_{\mathrm{EVPT}}\left(r_{1}, \ldots, r_{M}\right) .
\end{aligned}
$$

For the one case considered below where $E_{0}$ was defined selfconsistently, the last term in Eq. (48) was neglected and $\rho_{0}^{0}$ was replaced by $\rho_{0}$ in Eq. (50).

Properties are calculated using Eqs. (43) and (50). Finally, we note that an expression identical to Eq. (50) holds for the fixed coefficient (or frozen $\Psi_{0}$ ) VPT properties. A significant difference exists in the application of this expression to the two types of wave functions, however. In VPT the orthogonal complement configurations of $\Psi_{0}$ in the reference space are included in the $Q$ space and contribute to $\Psi_{1}$, and the intermediate normalization applied sets the coefficient of $\Psi_{0}$ to one. In QDVPT all linear combinations of the reference space functions are included in the $\mathbf{P}$ space, thus $\left\langle\Psi_{1} \mid \Psi_{1}\right\rangle$ contains no contributions from the reference space. In addition, the intermediate normalization applied has the sum of the squares of all reference space configurations equal to unity.

\section{RESULTS}

All calculations were performed with the MELDF suite of electronic structure codes from this laboratory. ${ }^{33}$ The $\mathrm{BeH}_{2},{ }^{34} \mathrm{CH}_{2}{ }^{2(\mathrm{c})}$ and ethylene ${ }^{35}$ basis sets and geometries were those used in Ref. 18 to allow comparison with previous full $\mathrm{Cl}$ results. ${ }^{2(\mathrm{c}), 2(\mathrm{~d}), 36}$ The $\mathrm{He}$ basis is the $3-12 \mathrm{G}$ basis of Binkley et al. ${ }^{37}$ In all cases except the ${ }^{1} B_{14}$ states of ethylene the MO basis sets were the SCF or two-configurational SCF (TCSCF) orbitals, with canonical virtual orbitals. For the ${ }^{1} B_{1 u}$ states of ethylene the average natural orbitals ${ }^{38}$ for the two lowest ${ }^{\prime} B_{1 u}$ states from a preliminary $C I$ were used as an MO basis. ${ }^{18,39,40}$ Properties are presented from multireference singles and doubles configuration interaction calculations (MRSDCI) (calculated as the expectation value of the given property operator), variational perturbation theory, QDVPT, and multireference linearized coupled-cluster wave functions. We have used second-order RayleighSchrödinger perturbation theory to select correlating configurations in some cases. ${ }^{18}$ Where this is done we also note the percentage of the second-order energy accounted for by the configurations retained in the variational part of the treatment. When perturbation theory selection was used we retained all single excitations relative to the reference functions.

We first present results based on QDVPT calculations on the systems examined in Ref. 18. Only for the ' $B_{1 u}$ states

\begin{tabular}{|c|c|c|c|c|c|}
\hline$r\left(\mathrm{BeH}_{2}\right)^{a}$ & $r\left(\mathrm{H}_{2}\right)^{: 3}$ & $\mathrm{TCSCF}^{b}$ & Corr $E^{*}$ & $\triangle \mathrm{MRSDCI}^{\prime}$ & $\triangle Q D V P T$ \\
\hline 1.00 & 4.16 & -15.7054 & 31.8 & 0.3 & -0.5 \\
\hline 2.00 & 3.24 & -15.6330 & 41.8 & 0.4 & -1.4 \\
\hline 2.50 & 2.78 & -15.5696 & 53.3 & 0.9 & -2.9 \\
\hline 2.75 & 2.55 & -15.5386 & 64.3 & 2.0 & -4.7 \\
\hline 3.00 & 2.32 & -15.5583 & 66.7 & 3.1 & -5.5 \\
\hline 3.50 & 1.86 & -15.6372 & 56.0 & 2.1 & -2.3 \\
\hline 4.0 & 1.4 & -15.6872 & 50.4 & 2.5 & -0.8 \\
\hline 6.0 & 1.4 & -15.7107 & 50.2 & 1.6 & -1.8 \\
\hline
\end{tabular}
of ethylene did we perform an iteration of the QDVPT equations to redefine $E_{0}$. In all other cases the results were based on using the appropriate eigenvalue of $H_{0}$ in the reference space as $E_{0}$.

In Table I results are presented from calculations at sev-

TABLE I. BeH $\mathrm{B}_{2}$ two-reference (MRSDCI) and QDVPT results, $C_{2 r}$ geometries

"Distances in bohr. $r\left(\mathrm{BeH}_{2}\right)$ is the distance from Be to the $\mathrm{H}_{2}$ midpoint. $r\left(\mathrm{H}_{2}\right)$ is the $\mathrm{H}_{2}$ bond length.

"Energy of the two configuration SCF wave function, in hartree.

"Correlation energy, in millihartree, relative to the two configuration SCF energy, using the full CI energies of Ref. 36.

"Error relative to the full Cl energies of Ref. 36, in millihartree. (MRSDCI) designates singles and doubles CI results [in this case a two-reference (MRSDCI)] and QDVPT denotes uniterated quasidegenerate variation. al perturbation theory results. 
TABLE II. MRSDCI and MRLCC, VPT, and QDVPT results for $\mathrm{CH}_{2}, C_{2 V}$ symmetry.

\begin{tabular}{ccccccc}
\hline \hline State & Refs. $^{a}$ & $\Delta$ MRSDCI $^{\mathrm{b}}$ & $\Delta$ MRLCC $^{\mathrm{b}}$ & $\Delta \mathrm{VPT}$ & $\Delta$ QDVPT $^{\mathrm{b}}$ & Full CI $^{\mathrm{c}}$ \\
\hline $1^{3} B_{1}$ & 1 & 4.7 & & & $-1.3^{\mathrm{d}}$ & -39.0463 \\
$1^{\prime} A_{1}$ & 1 & 8.9 & & & $-3.0^{\mathrm{d}}$ & -39.0272 \\
$1^{1} A_{1}$ & 2 & 5.0 & -1.3 & -1.3 & -1.3 & \\
$1^{\prime} A_{1}$ & 20 & 1.7 & -1.3 & -1.7 & -1.7 & \\
$1^{\prime} A_{1}$ & 29 & 1.1 & -1.2 & -1.6 & -1.6 & \\
\hline \hline
\end{tabular}

${ }^{a}$ The number of spin-adapted configurations in the reference space.

${ }^{b}$ Errors relative to the full $\mathrm{CI}$, in millihartree. The labels are defined in Table I.

${ }^{\mathrm{C}}$ Results from Ref. 2(c), in hartree.

'In the one-reference case, the MRLCC, VPT, and QDVPT methods are equivalent.

eral geometries of $\mathrm{BeH}_{2}$. In Ref. 18 it was shown that while MRLCC and VPT generally gave good agreement with full $\mathrm{CI}$ energies, the geometry $r\left(\mathrm{BeH}_{2}\right)=2.75 a_{0} r\left(\mathrm{H}_{2}\right)=2.55$ $a_{0}$ was particularly troublesome for either method. The VPT results gave a grossly incorrect ratio of the coefficients of the two reference functions in the final wave function, and the error in the total energy changed sign relative to the preceding or following points in the series, leading to a bumpy surface. A smooth surface and accurate relative sizes of the coefficients of the zeroth-order configurations were only recovered when the reference space size was increased significantly. MRLCC gave a smooth surface but because the reference space coefficients were taken from diagonalizing $H_{0}$, their ratio in the final wave function was also incorrect. It is seen that the uniterated QDVPT results compare favorably with MRSDCI (relative to the full CI energies). The ratio of the coefficients of the two reference functions ( $x$ in Ref. 18) was found to be -0.89 for QDVPT, -0.82 for MRSDCI, -0.57 for MRLCC, and +0.04 for VPT. The full CI value is -0.85 , indicating that QDVPT is capable of treating the strong mixing between the two zeroth-order functions much more easily than VPT.

In Table II we present results from MRSDCI, VPT, MRLCC, and QDVPT calculations on $\mathrm{CH}_{2}$, and compare them to the full CI results of Bauschlicher and Taylor. ${ }^{2(c)}$ It is seen that excellent agreement is obtained.

TABLE III. Total energies for $\mathrm{CH}_{2} \mathrm{He}_{n}, \mathrm{C}_{2 V}$ symmetry.

\begin{tabular}{|c|c|c|c|c|c|c|c|}
\hline$n$ & State & Refs." & \% PTK $^{b}$ & $\triangle \mathrm{MRSDCI}^{c}$ & $\Delta(\mathrm{MR}) \mathrm{LCC}^{\mathrm{c}}$ & $\triangle \mathrm{VPT}^{c}$ & QDVPT \\
\hline 0 & $1^{3} B_{1}$ & 1 & 100 & 4.7 & & -1.3 & \\
\hline 0 & $1^{\prime} A_{1}$ & 1 & 100 & 8.9 & & -3.0 & \\
\hline 0 & $1^{\prime} A_{1}$ & 2 & 100 & 5.0 & -1.3 & -1.3 & -1.3 \\
\hline 1 & $1^{3} B_{1}$ & 1 & 100 & 5.7 & & -1.4 & \\
\hline 1 & $1^{\prime} A_{1}$ & 1 & 100 & 10.3 & & -3.0 & \\
\hline 1 & $1^{\prime} A_{1}$ & 2 & 100 & 6.1 & -1.3 & -1.3 & -1.3 \\
\hline 3 & $1{ }^{3} B_{1}$ & 1 & 100 & 8.0 & & -1.5 & \\
\hline 3 & $1^{\prime} A_{1}$ & 1 & 100 & 13.2 & & -3.1 & \\
\hline 3 & $1^{\prime} A_{t}$ & 2 & 100 & 8.4 & -1.4 & -1.4 & -1.4 \\
\hline 5 & $1{ }^{3} B_{1}$ & 1 & 100 & 10.5 & & -1.6 & \\
\hline 5 & $1^{\prime} A_{1}$ & 1 & 100 & 16.3 & & -3.2 & \\
\hline 5 & $1^{\prime} A_{1}$ & 2 & $>99.9$ & 11.0 & -1.6 & -1.6 & -1.6 \\
\hline 9 & $1{ }^{3} B_{1}$ & 1 & $>99.9$ & 16.4 & & -1.7 & \\
\hline 9 & $1^{\prime} A_{1}$ & 1 & $>99.9$ & 23.2 & & -3.4 & \\
\hline 9 & $1^{\prime} A_{1}^{\prime}$ & 2 & $>99.9$ & 17.1 & -1.7 & -1.7 & -1.7 \\
\hline 12 & $1^{1} A_{1}$ & 2 & $>99.9$ & 22.2 & -1.8 & -1.7 & -1.8 \\
\hline 13 & $1{ }^{3} B_{1}$ & 1 & $>99.9$ & 23.3 & & -1.9 & \\
\hline 13 & $1^{\prime} A_{1}$ & 1 & $>99.9$ & 31.0 & & -3.6 & \\
\hline 13 & $1^{\prime} A_{1}$ & 2 & $>99.9$ & 24.0 & -1.9 & -1.9 & -1.9 \\
\hline 17 & $1^{3} B_{1}$ & 1 & $>99.9$ & 31.2 & & -2.1 & \\
\hline 17 & $1^{\prime} A_{1}$ & 1 & $>99.9$ & 39.7 & & -3.8 & \\
\hline 17 & $1^{\prime} A_{l}^{\prime}$ & 2 & $>99.9$ & 32.1 & -2.1 & -2.1 & -2.1 \\
\hline 25 & $1^{3} B_{1}$ & 1 & $>99.9$ & 49.8 & & -2.5 & \\
\hline 25 & $1^{\prime} A_{1}$ & 1 & $>99.9$ & 59.5 & & -4.1 & \\
\hline 25 & $1^{\prime} A_{1}$ & 2 & $>99.9$ & 50.8 & -2.5 & -2.5 & -2.5 \\
\hline
\end{tabular}

"The number of spin-adapted configurations in the reference space. In the one-reference case, the LCC, QDVPT, and VPT methods are equivalent.

${ }^{\mathrm{D}}$ The percentage of the second-order energy accounted for by the configurations treated variationally. A value of 100 indicates all single and double excitations were included. All single excitations from the reference functions were included in all cases.

"Errors relative to the full CI [Ref. 2(c)] in millihartree. (MR) LCC denotes a (multireference) linearized coupled-cluster calculation. VPT denotes a variational perturbation theory calculation. The remaining labels are defined in Table $I$ and the text. 
TABLE IV. Results from correlated treatments for $\mathrm{C}_{2} \mathrm{H}_{4}, " D_{2 h}$ symmetry.

\begin{tabular}{lccrcccc}
\hline \hline State & Refs. $^{b}$ & PTK $^{\mathrm{c}}$ & Conf. $^{\mathrm{d}}$ & MRSDCI & MRLCC & VPT & QDVPT \\
\hline $1^{\prime} A_{g}$ & 1 & 100 & 5252 & -78.3272 & $\ldots$ & -78.3615 & $\ldots$ \\
$1^{\prime} A_{g}$ & 4 & 100 & 23762 & -78.3330 & -78.3616 & -78.3618 & -78.3617 \\
$1^{\prime} A_{g}$ & 4 & 96.5 & 3242 & -78.3319 & -78.3563 & -78.3610 & -78.3592 \\
$1^{\prime} A_{g}$ & 2 & 100 & 10226 & -78.3356 & -78.3610 & -78.3622 & -78.3616 \\
$1^{\prime} B_{1 u}$ & $2^{2}$ & 100 & 21062 & -78.0287 & -78.0614 & -78.0614 & -78.0614 \\
$2^{\prime} B_{1 u}$ & $2^{e}$ & 100 & 21062 & -77.9911 & -78.0206 & -78.0206 & -78.0206 \\
\hline \hline
\end{tabular}

"All energies in hartree. The labels are defined in Tables I and III

${ }^{\mathrm{b}}$ Number of spin-adapted configurations in the reference space. For the ${ }^{\prime} B_{1}$ states the average NOs were used (see the text) as an MO basis. For the two-reference 1 ' $A_{g}$ calculation the TCSCF orbitals were used. For the remaining $1^{\prime} A_{x}$ calculations the one-reference SCF orbitals were used.

'Percentage of the second-order Rayleigh-Schrödinger perturbation theory energy accounted for by the configurations retained. All single excitations were kept.

d Number of spin-adapted configurations treated beyond second-order Rayleigh-Schrödinger perturbation theory.

'The choice of $E_{0}$ for the QDVPT equation was iterated once. The coefficients defining $\psi_{0}$ for MRLCC and VPT were chosen to be the coefficients of the reference space configurations used to define $E_{0}$ in the final iteration of the QDVPT equations.

In Table III we present results from MRSDCI, MRLCC, VPT, and QDVPT calculations on the model system $\mathrm{CH}_{2} \mathrm{He}_{n}$. The $\mathrm{He}$ are placed at $10 a_{0}$ intervals along the $+z$ axis (defined as the symmetry axis of $\mathrm{CH}_{2}, \mathrm{C}$ is at the origin and the $\mathrm{H}$ atoms lie in $-z$ half-plane). The nearest $\mathrm{He}$ is $10 a_{0}$ from the $\mathrm{C}$. Since we have used the same basis for
$\mathrm{CH}_{2}$ as in the results of Table II and since the He are well separated from each other and from $\mathrm{CH}_{2}$, we can calculate the full $\mathrm{CI}$ energy for this system from the results of Ref. 2 (c) and full Cl on $\mathrm{He}\left(E_{\mathrm{He}}=-2.8505767\right.$ hartree). The energy values for the various methods are reported relative to the computed full $\mathrm{CI}$ values (based on the expression

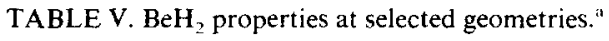

\begin{tabular}{|c|c|c|c|c|c|c|c|c|c|}
\hline$r\left(\mathrm{BeH}_{2}\right)$ & $r\left(\mathbf{H}_{2}\right)$ & Refs. & Quantity & Ref. space ${ }^{b}$ & MRSDCI & MRLCC & VARPT & QDVPT & Full CI \\
\hline \multirow[t]{4}{*}{1.0} & 4.16 & 2 & $\langle z\rangle$ & 0.794 & 0.686 & 0.674 & 0.671 & 0.671 & 0.689 \\
\hline & & & $\left\langle x^{2}\right\rangle$ & 4.80 & 4.84 & 4.84 & 4.85 & 4.85 & 4.84 \\
\hline & & & $\langle z\rangle_{\mathrm{fix}}$ & & & 0.674 & 0.671 & & \\
\hline & & & $\left\langle x^{2}\right\rangle_{\text {fix }}$ & & & 4.84 & 4.85 & & \\
\hline \multirow[t]{4}{*}{3.5} & 1.86 & 2 & $\langle z\rangle$ & -1.011 & -0.907 & -0.847 & & -0.859 & -0.889 \\
\hline & & & $\left\langle x^{2}\right\rangle$ & 7.51 & 7.48 & 7.44 & 7.46 & 7.46 & 7.47 \\
\hline & & & $\langle z\rangle_{\text {fix }}$ & & & -0.829 & -0.863 & & \\
\hline & & & $\left\langle x^{2}\right\rangle_{\mathrm{fix}}$ & & & 7.38 & 7.48 & & \\
\hline \multirow[t]{4}{*}{6.0} & 1.4 & 2 & $\langle z\rangle$ & -0.093 & -0.079 & -0.073 & -0.073 & -0.073 & -0.077 \\
\hline & & & $\left\langle x^{2}\right\rangle$ & 7.02 & 6.95 & 6.92 & 6.93 & 6.92 & 6.94 \\
\hline & & & $\langle z\rangle_{\mathrm{fix}}$ & & & -0.072 & -0.073 & & \\
\hline & & & $\left\langle x^{2}\right\rangle_{\text {fix }}$ & & & 6.88 & 6.93 & & \\
\hline \multirow{4}{*}{2.0} & 3.24 & 2 & $\langle z\rangle$ & 1.48 & 1.26 & 1.23 & 1.21 & 1.21 & 1.26 \\
\hline & & & $\left\langle x^{2}\right\rangle$ & 5.05 & 5.08 & 5.09 & 5.10 & 5.10 & 5.09 \\
\hline & & & $\langle z\rangle_{\mathrm{fix}}$ & & & 1.23 & 1.21 & & \\
\hline & & & $\left\langle x^{2}\right\rangle_{f i x}$ & & & 5.08 & 5.10 & & \\
\hline \multirow[t]{5}{*}{2.0} & $3.24^{\circ}$ & 9 & Energy & & -15.6748 & -15.6745 & -15.6750 & -15.6749 & $\ldots$ \\
\hline & & & $\langle z\rangle$ & 1.43 & 1.26 & 1.28 & 1.25 & 1.25 & $\ldots$ \\
\hline & & & $\left\langle x^{2}\right\rangle$ & 5.07 & 5.09 & 5.08 & 5.09 & 5.09 & $\cdots$ \\
\hline & & & $\langle z\rangle_{\mathrm{fix}}$ & & & 1.28 & 1.25 & & \\
\hline & & & $\left\langle x^{2}\right\rangle_{\mathrm{fix}}$ & & & 5.08 & 5.09 & & \\
\hline \multirow[t]{4}{*}{3.0} & 2.32 & 2 & $\langle z\rangle$ & -1.40 & -1.20 & -0.954 & -0.948 & -0.952 & -1.16 \\
\hline & & & $\left\langle x^{2}\right\rangle$ & 7.68 & 7.64 & 7.51 & 7.50 & 7.50 & 7.63 \\
\hline & & & $\langle z\rangle_{\text {fix }}$ & & & -0.955 & -0.947 & & \\
\hline & $2.32^{c}$ & 9 & $\begin{array}{l}\left\langle x^{2}\right\rangle_{\text {iix }} \\
\text { Energy }\end{array}$ & & & 7.51 & & & \\
\hline \multirow{4}{*}{3.0} & $2.32^{2}$ & 9 & Energy & -139 & -15.6246 & -15.6267 & -15.6283 & -15.6281 & $\cdots$ \\
\hline & & & $\left\langle x^{2}\right\rangle$ & $\begin{array}{r}-1.39 \\
7.82\end{array}$ & $\begin{array}{r}-1.16 \\
762\end{array}$ & $\begin{array}{r}-1.14 \\
7.58\end{array}$ & $\begin{array}{r}-1.05 \\
754\end{array}$ & & $\cdots$ \\
\hline & & & $\langle z\rangle$ & & & $\begin{array}{r}1.14 \\
-114\end{array}$ & $\begin{array}{r}1.04 \\
-104\end{array}$ & & $\cdots$ \\
\hline & & & $\left\langle x^{2}\right\rangle_{\text {fix }}$ & & & 7.59 & 7.51 & & \\
\hline
\end{tabular}

"All quantities in atomic units. The various methods are defined in Sec. II and the labels are defined in Tables I and III. Properties labeled fix for MRLCC and

VPT are calculated using Eq. (36), otherwise Eqs. (34) and (35) are used for VPT and MRLCC, respectively.

${ }^{\mathrm{h}}$ The value of the peoperty for the $\mathrm{CI}$ within the reference space.

'The reference functions were the dominant spin-adapted configurations in the QDVPT calculation at the given geometry. 
$E_{\text {full }}^{A}+n E_{\mathrm{He}}$, where $A$ labels the state of interest). The MRSDCI results, as expected, begin to deviate significantly from the full $\mathrm{CI}$ energies as the number of $\mathrm{He}$ is increased. The MRLCC, VPT, and QDVPT results are all significantly closer to the full CI values as $n$ is increased, but they show some drift away from the full CI values. However, close inspection shows this drift with $n$ is essentially linear. This drift is due to the fact that the total energy for these methods should vary as $E_{\text {Meth }}^{A}+n\left(E_{\mathrm{He}}\right)_{\mathrm{LCC}}$, since for the reference spaces chosen (the reference functions involve excitations localized on $\mathrm{CH}_{2}$ ) the He are treated at the single reference level. All three methods reduce to the linearized coupled cluster (LCC) method for a single reference function. Thus the change in $\Delta E$ with increasing He for MRLCC, VPT, and QDVPT goes as $n\left[\left(E_{\mathrm{He}}\right)_{\mathrm{LCC}}-E_{\mathrm{He}}\right]$.

In Table IV results of (MRSDCI), MRLCC, VPT, and QDVPT calculations are presented for various states of ethylene. The QDVPT results are in good agreement with the MRLCC and VPT results. For the ${ }^{1} B_{1 u}$ states the QDVPT equations were iterated once to redefine $E_{0}$, since the final $C_{P}$ were quite different from those obtained by diagonalizing $H_{0}$ over the reference space. The VPT and MRLCC results are based on the coefficients of the QDVPT reference space functions used in the final iteration of the QDVPT equations. The energy values based on these reference functions are quite close to those reported previously where a somewhat different definition of $\Psi_{0}$ was used. ${ }^{18}$ The excitation energies based on these results are in good agreement with size-consistency corrected $\mathrm{CI}$ values. ${ }^{18}$

We next examine the results of calculations of one-elec- tron properties for these wave functions. The results from the calculations on $\mathrm{BeH}_{2}$ are given in Tables $\mathrm{V}$ and VI. For VPT and MRLCC we present both fixed coefficient properties [Eq. (35)] and properties calculated where the coefficients defining $\Psi_{0}$ are allowed to change with application of the field [Eqs. (33) and (34) ]. Several geometries ${ }^{36}$ are examined in Table $\mathrm{V}$ for treatments based on two-configuration SCF wave functions. Representative examples for the effects of expansion of the reference space are also given in Table V. Similar results were obtained at other geometries. The full CI property results were obtained in the present study. For the geometries in Table V, the singles and doubles CI results are somewhat closer to the full CI results than MRLCC, VPT, or QDVPT, but in general the agreement for all the methods is quite good. Expansion of the reference space leads to better agreement for the MRLCC, VPT, and QDVPT results. In all cases the fixed coefficient MRLCC or VPT results are not as accurate as the variable coefficient results.

In Table VI results are shown from calculations at a particularly difficult geometry. In the two-reference case the MRLCC and QDVPT energies and properties are reasonably close to the full CI results, but the VPT error in the properties is quite large. Note that the fixed coefficient properties are generally worse. Expansion of the reference space leads to better agreement for all methods, with the QDVPT results converging to the full $\mathrm{CI}$ values somewhat quicker.

Results from calculations on $\mathrm{CH}_{2} \mathrm{He}_{n}(n=0, \ldots, 25)$ are shown in Table VII; full CI results for $n=0^{2(\mathrm{~d})}$ are listed as well. Since the He are well separated from each other and

TABLE VI. BeH, properties at $r\left(\mathrm{BeH}_{2}\right)=2.75 \mathrm{~b}, r\left(\mathrm{H}_{2}\right)=2.55 \mathrm{~b}^{\mathrm{a}}{ }^{\mathrm{a}}$

\begin{tabular}{|c|c|c|c|c|c|c|c|}
\hline Refs. & Quantity & Ref. space ${ }^{b}$ & MRSDCI & MRICC & VARPT & QDVPT & Full CI \\
\hline $2^{c}$ & $\begin{array}{l}\text { Energy } \\
\langle z\rangle \\
\left\langle x^{2}\right\rangle \\
\langle z\rangle_{\mathrm{fix}} \\
\left\langle x^{2}\right\rangle_{\mathrm{fix}}\end{array}$ & $\begin{array}{c}-15.5386 \\
0.807 \\
5.79\end{array}$ & $\begin{array}{c}-15.6009 \\
0.135 \\
6.30\end{array}$ & $\begin{array}{c}-15.6053 \\
0.191 \\
6.23 \\
0.592 \\
5.84\end{array}$ & $\begin{array}{c}-15.5986 \\
0.403 \\
6.14 \\
1.80 \\
4.80\end{array}$ & $\begin{array}{c}-15.6076 \\
-0.111 \\
6.50\end{array}$ & $\begin{array}{c}-15.6029 \\
0.090 \\
6.35\end{array}$ \\
\hline $15^{\mathrm{d}}$ & $\begin{array}{l}\text { Energy } \\
\langle z\rangle \\
\left\langle x^{2}\right\rangle \\
\langle z\rangle_{\mathrm{fix}} \\
\left\langle x^{2}\right\rangle_{\mathrm{fix}}\end{array}$ & $\begin{array}{c}-15.5788 \\
0.716 \\
5.91\end{array}$ & $\begin{array}{c}-15.6028 \\
0.100 \\
6.34\end{array}$ & $\begin{array}{c}-15.6010 \\
0.306 \\
6.12 \\
0.720 \\
5.75\end{array}$ & $\begin{array}{c}-15.6063 \\
-0.490 \\
6.92 \\
-0.990 \\
7.35\end{array}$ & $\begin{array}{c}-15.6037 \\
0.024 \\
6.41\end{array}$ & \\
\hline $12^{\mathrm{e}}$ & $\begin{array}{l}\text { Energy } \\
\langle z\rangle \\
\left\langle x^{2}\right\rangle \\
\langle z\rangle_{\mathrm{fix}} \\
\left\langle x^{2}\right\rangle_{\mathrm{fix}}\end{array}$ & $\begin{array}{c}-15.5814 \\
0.320 \\
6.22\end{array}$ & $\begin{array}{c}-15.6029 \\
0.093 \\
6.39\end{array}$ & $\begin{array}{c}-15.6026 \\
0.223 \\
6.23 \\
0.389 \\
6.06\end{array}$ & $\begin{array}{c}-15.6038 \\
-0.060 \\
6.48 \\
-0.207 \\
6.63\end{array}$ & $\begin{array}{c}-15.6033 \\
0.069 \\
6.37\end{array}$ & \\
\hline $17^{\mathrm{f}}$ & $\begin{array}{l}\text { Energy } \\
\langle z\rangle \\
\left\langle x^{2}\right\rangle \\
\langle z\rangle_{\mathrm{fix}} \\
\left\langle x^{2}\right\rangle_{\mathrm{fix}}\end{array}$ & $\begin{array}{c}-15.5850 \\
0.052 \\
6.47\end{array}$ & $\begin{array}{c}-15.6029 \\
0.090 \\
6.35\end{array}$ & $\begin{array}{c}-15.6028 \\
0.118 \\
6.33 \\
0.136 \\
6.31\end{array}$ & $\begin{array}{c}-15.6032 \\
0.083 \\
6.48 \\
0.055 \\
6.38\end{array}$ & $\begin{array}{c}-15.6031 \\
0.091 \\
6.35\end{array}$ & \\
\hline
\end{tabular}

"All quantities in atomic units. The various methods are defined in Sec. Il.

${ }^{b}$ Results from $\mathrm{Cl}$ over the reference space configurations.

"The two-reference configurations are from the two-configuration SCF wave function.

${ }^{d}$ The reference configurations are taken from the 15 dominant configurations (Ref. 41 ) in the two-configuration-based VPT calculation; their coefficients were obtained by diagonalizing $H$ over the reference space.

${ }^{\mathrm{C}}$ The reference configurations are taken from the 12 dominant configurations (Ref. 41 ) in the two-configuration-based MRSDCI calculation. The zerothorder wave function was defined by diagonalizing $H$ over the reference space.

'The reference configurations are taken from the 17 dominant configurations (Ref. 41 ) in the 12-configuration-based MRSDCI calculation. The zerothorder wave function was defined by diagonalizing $H$ over the reference space. 
TABLE VII. Results for $\mathrm{CH}_{2} \mathrm{He}_{n}:\langle z\rangle$ vs $n^{.4}$

\begin{tabular}{|c|c|c|c|c|c|c|c|}
\hline$n$ & State & Refs. & PTK $^{h}$ & MRSDCI & MRLCC & VARPT & QDVPT \\
\hline \multirow[t]{5}{*}{0} & $1^{3} B_{1}$ & 1 & 100 & -0.2630 & $\cdots$ & -0.2742 & $\cdots$ \\
\hline & $1^{\prime} A_{1}$ & 1 & 100 & -0.7538 & $\ldots$ & -0.6835 & $\ldots$ \\
\hline & $1^{\prime} A_{1}$ & 2 & 100 & -0.7232 & -0.7217 & -0.7232 & -0.7225 \\
\hline & $I^{\prime} A_{1}$ & 20 & 100 & -0.7192 & -0.7196 & -0.7124 & -0.7149 \\
\hline & $1^{\prime} A_{1}$ & 29 & 100 & -0.7177 & -0.7183 & -0.7113 & -0.7138 \\
\hline \multirow[t]{3}{*}{1} & ${ }^{3} B_{1}$ & 1 & 100 & -0.2622 & .. & -0.2742 & . \\
\hline & $1^{\prime} A_{1}$ & 1 & 100 & -0.7567 & $\ldots$ & -0.6835 & $\ldots$ \\
\hline & $1^{\prime} A$ & 2 & 100 & -0.7232 & -0.7217 & -0.7233 & -0.7225 \\
\hline \multirow[t]{3}{*}{3} & $1^{3} B_{1}$ & 1 & 100 & -0.2606 & $\ldots$ & -0.2742 & $\cdots$ \\
\hline & $1^{\prime} A_{1}$ & 1 & 100 & -0.7616 & $\cdots$ & -0.6835 & $\cdots$ \\
\hline & $1 ' A$ & 2 & 100 & -0.7231 & -0.7217 & -0.7236 & -0.7225 \\
\hline \multirow[t]{3}{*}{5} & $1^{3} B_{1}$ & 1 & 100 & -0.2593 & $\ldots$ & -0.2742 & $\ldots$ \\
\hline & $1^{\prime} A_{1}$ & 1 & 100 & -0.7658 & $\ldots$ & -0.6835 & $\ldots$ \\
\hline & $I^{\prime} A_{1}$ & 2 & 99.9 & -0.7231 & -0.7217 & -0.7241 & -0.7225 \\
\hline \multirow[t]{3}{*}{9} & $1^{3} B_{1}$ & 1 & 99.9 & -0.2569 & $\ldots$ & -0.2739 & $\ldots$ \\
\hline & $1^{\prime} A_{1}$ & 1 & 99.9 & -0.7720 & $\cdots$ & -0.6832 & .. \\
\hline & $1^{\prime} A_{1}$ & 2 & 99.9 & -0.7229 & -0.7217 & -0.7275 & -0.7225 \\
\hline 12 & $1^{\prime} A_{1}$ & 2 & 99.9 & -0.7227 & -0.7217 & -0.6431 & -0.7225 \\
\hline \multirow[t]{3}{*}{13} & $1^{3} B_{1}$ & 1 & 99.9 & -0.2552 & .. & -0.2739 & $\cdots$ \\
\hline & $1 A$ & 1 & 99.9 & -0.7768 & $\ldots$ & -0.6832 & $\ldots$ \\
\hline & $1^{\prime} A_{1}^{\prime}$ & 2 & 99.9 & -0.7226 & -0.7217 & -0.7076 & -0.7225 \\
\hline \multirow[t]{3}{*}{17} & ${ }^{3} B_{1}$ & 1 & 99.9 & -0.2538 & .. & -0.2739 & $\ldots$ \\
\hline & $I^{\prime} A_{1}$ & 1 & 99.9 & -0.7805 & $\cdots$ & -0.6832 & $\cdots$ \\
\hline & $1^{\prime} A_{1}$ & 2 & 99.9 & -0.7224 & -0.7217 & -0.7185 & -0.7224 \\
\hline \multirow[t]{3}{*}{25} & $1^{3} B_{1}$ & 1 & 99.9 & -0.2516 & $\cdots$ & -0.2739 & $\cdots$ \\
\hline & $1^{\prime} A_{1}$ & 1 & 99.9 & -0.7858 & $\ldots$ & -0.6832 & $\ldots$ \\
\hline & ${ }^{\prime} A_{1}$ & 2 & 99.9 & -0.7219 & -0.7217 & -0.7205 & -0.7224 \\
\hline
\end{tabular}

"The labels are defined in Tables I and III. Properties are reported in atomic units. The orbitals used for the multireference calculations on the $1{ }^{\prime} A_{1}$ state are TCSCF orbitals. The SCF values of $\langle z\rangle$ are ${ }^{3} B_{1}:-0.243 ;{ }^{\prime} A_{1}$ (one-reference): $-0.807 ;{ }^{\prime} A_{1}$ (two-reference): -0.716 ; the full CI values [Ref. 2 (d) ] are ${ }^{3} B_{1}:-0.264 ;{ }^{\prime} A_{1}:-0.716$.

${ }^{\mathrm{h}}$ The percentage of the second-order perturbation theory energy lowering accounted for by the configurations treated variationally. A value of 100 indicates that no perturbation theory selection was performed. For the two-reference cases PT selection was performed on both roots of the zeroth-order space.

from $\mathrm{CH}_{2}$, and each $\mathrm{He}$ makes no contribution to the dipole moment, the full CI dipole moments for $n \neq 0$ would be identical to the $n=0$ results. In a one-reference wave function MRLCC, VPT, and QDVPT are all equivalent. For the $1^{\prime} A_{1}$ state all methods are sensitive to the expansion of the reference space from one function to two, with significantly better agreement obtained in the two-reference case. The VPT result for the one-reference case leads to an error comparable to the one-reference CI wave function. For $n=0$ we also present results from expanded reference space calculations on the $1^{\prime} A_{1}$ state, based on the TCSCF orbitals with canonical virtual orbitals. It is seen that all methods approach the full CI values, although only the CI and MRLCC results appear to converge monotonically.

For $n>0$ it is seen that the one-reference ${ }^{3} B_{1}$ value of $\langle z\rangle$ for the CI wave function changes as the number of He atoms is increased, whereas the VPT value does not. Note that use of perturbation theory selection in VPT alters the computed value of the property by 0.0003 for the ${ }^{3} B_{1}$ state. The tworeference $1^{\prime} A_{1} \mathrm{CI}\langle z\rangle$ changes slowly over the range of $n$ considered here. In part this slow change is due to the small difference between the TCSCF value of $\langle z\rangle$ (to which the MRSDCI value goes as $n$ increases) and the MRSDCI value. The MRLCC and QDVPT results are quite stable as $n$ is increased, the small change in $\langle z\rangle$ for QDVPT arising from the size inconsistency discussed above. The error in the VPT result, which is large even by $n=9$, arises from a crossing of
$E_{0}$ by one of the eigenvalues of $\mathbf{Q} H \mathbf{Q}$ between $n=9$ and 13 . In addition to the odd behavior of $\langle z\rangle$, corroborating evidence that this crossing is occurring comes from comparison of the total VPT and MRLCC energies. In Ref. 18 it was shown that $E_{\mathrm{VPT}}$ is less than or equal to $E_{\mathrm{MRLCC}}$ whenever $E_{0}$ is the lowest eigenvalue of $H_{0}$ (i.e., when the eigenvalues of $\mathbf{Q} H \mathbf{Q}$ are all greater than $E_{0}$ ). We have found that at $n=12$ the MRLCC total energy becomes lower than the VPT energy, suggesting that a crossing has occurred. It is interesting to note that the results of Table III show that this crossing has no significant effect on the total VPT energy. Thus, the size inconsistency in VPT manifests itself in the one-electron properties to a much greater extent than in the total energy.

Results obtained for various states of ethylene are shown in Table VIII. Examining the $1^{\prime} A_{g}$ results it is seen that all four methods are sensitive to the expansion of the reference space, especially in the two-reference case, where the MOs are from a two-configuration SCF. The fixed and variable coefficient MRLCC and VPT properties agree to the number of significant figures reported. In the ${ }^{1} B_{1 u}$ cases, the CI results differ from the QDVPT, VPT, or MRLCC results to a greater extent. The QDVPT, MRLCC, and VPT values of $\left\langle x^{2}\right\rangle$ for the $1^{\prime} B_{1 u}$ state are somewhat smaller than the $C I$ value. For the $2^{\prime} B_{1}$ state the QDVPT, MRLCC, and VPT values are all somewhat larger than the CI values. The coefficients defining $\Psi_{0}$ for the ${ }^{1} B_{1 u}$ states in the VPT 
TABLE VIII. $\mathrm{C}_{2} \mathrm{H}_{4}$ properties. $^{a}$

\begin{tabular}{|c|c|c|c|c|c|c|c|}
\hline State & Refs. & PTK $^{\mathrm{b}}$ & Quantity & SDCI & MRLCC & VARPT & QDVPT \\
\hline $1^{1} A_{g}$ & 1 & 100 & $\begin{array}{c}\left\langle x^{2}\right\rangle \\
\left\langle y^{2}\right\rangle+\left\langle z^{2}\right\rangle\end{array}$ & $\begin{array}{l}11.8 \\
18.0\end{array}$ & $\begin{array}{l}\cdots \\
\cdots\end{array}$ & $\begin{array}{l}11.6 \\
18.1\end{array}$ & $\begin{array}{l}\cdots \\
\cdots\end{array}$ \\
\hline $1^{\prime} A_{g}$ & 4 & 100 & $\begin{array}{c}\left\langle x^{2}\right\rangle \\
\left\langle y^{2}\right\rangle+\left\langle z^{2}\right\rangle \\
\left\langle x^{2}\right\rangle \\
\left(\left\langle y^{2}\right\rangle+\left\langle z^{2}\right\rangle\right)_{\mathrm{fix}}\end{array}$ & $\begin{array}{l}11.8 \\
18.1\end{array}$ & $\begin{array}{l}11.6 \\
18.2 \\
11.6 \\
18.2\end{array}$ & $\begin{array}{l}11.6 \\
18.2 \\
11.6 \\
18.2\end{array}$ & $\begin{array}{l}11.6 \\
18.2\end{array}$ \\
\hline $1^{\prime} A_{g}$ & $2^{\mathrm{c}}$ & 100 & $\begin{array}{c}\left\langle x^{2}\right\rangle \\
\left\langle y^{2}\right\rangle+\left\langle z^{2}\right\rangle \\
\left\langle x^{2}\right\rangle\end{array}$ & 11.7 & $\begin{array}{l}11.7 \\
18.2 \\
11.7\end{array}$ & $\begin{array}{l}11.7 \\
18.2 \\
11.7\end{array}$ & 11.7 \\
\hline $1{ }^{3} B_{1 u^{e}}$ & 1 & $>99$ & $\begin{array}{c}\left(\left\langle y^{2}\right\rangle+\left\langle z^{2}\right\rangle\right)_{\mathrm{fix}} \\
\left\langle x^{2}\right\rangle \\
\left\langle y^{2}\right\rangle+\left\langle z^{2}\right\rangle\end{array}$ & $\begin{array}{l}18.1 \\
11.8 \\
19.6\end{array}$ & $\begin{array}{c}18.2 \\
\cdots \\
\cdots\end{array}$ & $\begin{array}{l}18.2 \\
11.9 \\
19.8\end{array}$ & 18.2 \\
\hline $1^{\prime} B_{1 u}^{e}$ & $2^{\mathrm{d}}$ & 100 & $\begin{array}{c}\left\langle x^{2}\right\rangle \\
\left\langle y^{2}\right\rangle+\left\langle z^{2}\right\rangle\end{array}$ & $\begin{array}{l}27.8 \\
38.7\end{array}$ & $\begin{array}{l}23.7 \\
33.4\end{array}$ & $\begin{array}{l}23.7 \\
33.5\end{array}$ & $\begin{array}{l}23.3 \\
32.9\end{array}$ \\
\hline $2^{\prime} B_{1 u}{ }^{e}$ & $2^{d}$ & 100 & $\begin{array}{c}\left\langle x^{2}\right\rangle \\
\left\langle y^{2}\right\rangle+\left\langle z^{2}\right\rangle\end{array}$ & $\begin{array}{l}59.1 \\
80.0\end{array}$ & $\begin{array}{l}70.6 \\
94.8\end{array}$ & $\begin{array}{l}70.5 \\
94.8\end{array}$ & $\begin{array}{l}70.6 \\
94.8\end{array}$ \\
\hline
\end{tabular}

${ }^{a}$ All quantities in atomic units. The various methods are defined in Sec. II. Calculations based on Hartree-Fock molecular orbitals unless otherwise noted.

${ }^{b}$ Denotes the percentage of the second-order Rayleigh-Schrödinger perturbation theory energy lowering corresponding to the configurations treated variationally.

${ }^{\mathrm{c}}$ The molecular orbitals are from a two-configuration SCF that correlates the $\pi$ electrons.

${ }^{\mathrm{d}}$ The molecular orbitals are the average natural orbitals from a preliminary Cl calculation (see the results section). The coefficients defining $\Psi_{6}$ for VPT and MRLCC were those used to define $E_{0}$ in the final QDVPT iteration. The definition of $E_{0}$ in the QDVPT results was obtained via the iterative process described in the text.

${ }^{\mathrm{e}}$ Since the zeroth-order wave functions are defined iteratively, and not as the eigenfunctions of $H_{o}^{f}$, the $B_{1 u}$ properties are evaluated using Eq. (36) for MRLCC and VPT, and the final term in Eq. (48) is neglected for the QDVPT properties.

and MRLCC calculations were those used in the final iteration of the QDVPT equations.

\section{DISCUSSION}

Comparison of the VPT results of Ref. 18 and the QDVPT results presented above suggests that QDVPT and VPT are comparable whenever the effective coupling in the reference space is small and/or no instabilities are present in the VPT equations due to crossings of an eigenvalue of QHQ and $E_{0}$. However, when such instabilities exist for VPT, or when the effective coupling is large, QDVPT remains accurate. The elimination of these problems occurs because, relative to the VPT equations, $E_{0}$ has been replaced by $E_{\text {Total }}$ in the diagonal elements corresponding to the orthogonal complement reference space configurations. $E_{0}$ is retained on the diagonal for the single and double excitations in QDVPT, but since the single and double excitations do not correlate each other to a significant extent, no crossings are expected of the eigenvalues of $\mathbf{Q} H \mathbf{Q}$ with $E_{0}$ for QDVPT.

Even with the problems noted above for VPT, it is seen in the results on the $\mathrm{CH}_{2} \mathrm{He}_{n}$ systems that VPT, MRLCC, and QDVPT all yield more nearly size-consistent total energies than does MRSDCI. MRLCC is strictly size consistent for this choice of reference space.

Concerning the properties results the agreement obtained between the CI, MRLCC, VPT, and QDVPT results is quite good. For $\mathrm{BeH}_{2}, \mathrm{CH}_{2}$, and most states of ethylene we find agreement to within a few percent of the total value of the property. Based on the $\mathrm{BeH}_{2}$ results and the $\mathrm{CH}_{2}{ }^{3} B_{1}$ one-reference results it can be seen that the $\mathrm{CI}$ properties tend to be more rapidly convergent than the VPT, MRLCC, and QDVPT properties. However, increasing the reference space from two to nine functions for the $\mathrm{BeH}_{2}$ example brought the VPT, MRLCC, and QDVPT results into much closer agreement with the CI results. Similar results were found for the $1^{\prime} A_{1}$ state of $\mathrm{CH}_{2}$ on going from a one- to a multireference based treatment.

The possible sensitivity of the calculated properties to the choice of $\Psi_{0}$ is graphically illustrated by the results of Table VI. It is seen that the energy estimate obtained by MRLCC, QDVPT, or VPT can be reasonably accurate (error $<4$ mhartree), but that the error in the property estimate can still be quite large. For MRLCC and VPT a significant expansion of the reference space was required to obtain good agreement with the full CI property results, and even still the properties were not as accurate as the MRSDCI or QDVPT values. QDVPT gave reasonable results with somewhat less effort. It should be noted that this was also a difficult geometry for the two-reference singles and doubles CI approach, and expansion of the reference space was again required to obtain good agreement with the full CI. However, the error for a given reference space was found to be smaller for the MRSDCI results than the MRLCC, VPT, or QDVPT.

The results for the $\mathrm{CH}_{2} \mathrm{He}_{n}$ system illustrate a number of interesting points. First, the value of $\langle z\rangle$ is dependent on the number of $\mathrm{He}$ atoms for the MRSDCI properties, while for MRLCC and QDVPT $\langle z\rangle$ was basically independent of $n$. The ratio of the MRSDCI error in $\langle z\rangle$ to the SCF error in $\left.\langle z\rangle\left[\Delta=\left(G_{\mathrm{SDCl}}-T_{\mathrm{Full}}\right) / G_{\mathrm{SCF}}-G_{\mathrm{Full}}\right)\right]$ changed considerably for the values of $n$ examined here. For the ${ }^{3} B_{1}$ state 
with $n=0 \Delta$ equals 0.05 , while for $n=25$ it was 0.57 . For the one reference description of the $1^{\prime} A_{1}$ state the change in the CI value of $\langle z\rangle$ is quite large, with $\Delta=0.30$ for $n=0$, while at $n=25 \Delta$ was 0.77 . The two configuration SCF value for $\langle z\rangle$ for the ${ }^{1} A_{1}$ state is closer to the full $\mathrm{CI}$ than any of the correlated treatments. However, it is still the case that an increase in $n$ changes the MRSDCI value of $\langle z\rangle$ significantly. It should be noted that the ${ }^{3} B_{1}$ MRSDCI values for $\langle z\rangle$ are closer to the full CI values than those of the perturbative treatments for all but the largest $n$.

The results of Table VII also highlight the limitations of VPT for multireference cases. It is seen that the error in the VPT value of $\langle z\rangle$ becomes unacceptable when $n$ is equal to 12 . With larger $n$ the value of $\langle z\rangle$ again becomes close to the full CI value, but this agreement is most likely fortuitous. However, since the VPT energy agrees with the MRLCC and QDVPT energies to within one in the fourth decimal place it is clear that the coefficients of the single and double excitations from $\Psi_{0}$ are similar in all three methods [ $\mathrm{cf}$. the energy expression, Eq. (7) ]. Due to the variational nature of these methods, the error in the energy goes quadratically with the error in $\Psi_{1}$, whereas the error in the property is first order in the error in $\Psi_{1}$. The main source of the error in $\langle z\rangle$ is an inordinately large coefficient for the configuration which is the orthogonal complement of $\Psi_{0}$. The total energy is basically unaffected because $\Psi_{0}$ is taken as the eigenfunction of $H$ in the reference space and $\left\langle\Psi_{c}|H| \Psi_{0}\right\rangle$ equals zero. Thus, while $\Psi_{c}$ can have no direct effect on the energy, it can still have quite a large effect on the properties.

In most cases the ethylene results are in good agreement for all of the methods examined. The QDVPT, MRLCC, and VPT wave functions predict that the $1^{\prime} B_{1 u}$ state is significantly more diffuse than either the ground state or the $1^{3} B_{1 u}$ state, in agreement with CI. All three methods show similar sensitivity in the properties to expansion of the reference space, whereas the CI energies are much more sensitive to reference space expansion than the VPT or MRLCC energies. The MRLCC, QDVPT, and VPT results predict the $1^{1} B_{1 u}$ state to be less diffuse than the MRSDCI result. Previous MCSCF calculations on this state ${ }^{42}$ have found similar results and it was suggested that size inconsistency in the MRSDCI may be the cause of the discrepancy. Our results lend support to this suggestion, but must be viewed with some caution. In the VPT, MRLCC, and QDVPT calculations reported where we have neglected the terms in the property expressions [Eqs. (35) or (36) and (45) or (50) and (45) ] related to derivatives of the reference space coefficients with respect to perturbation strength. Since for the ${ }^{1} B_{1 u}$ states the reference space coefficients were defined iteratively and not as the eigenfunctions of the zeroth-order Hamiltonian, these terms will, in general, be nonzero. We expect them to be small, but further calculations are required to assess this. Similar comments apply to the $2{ }^{\prime} B_{1 u}$ state, which VPT, MRLCC, and QDVPT predict to be more diffuse than the MRSDCI result. Larger CI, QDVPT, VPT, and MRLCC results for these states are required to answer this question.

The question arises as to which of the three methods (other than CI) is expected to be the most reliable. We be- lieve that QDVPT should generally be the most accurate method because it treats the interaction of the reference functions to infinite order, thus avoiding the numerical instabilities of VPT, while still allowing the weights of the reference configurations to change in the final wave function, unlike MRLCC. However, the results presented above suggest that both MRLCC and QDVPT can be quite accurate and should be useful for large systems where size-inconsistency effects in CI results are of the same order as the errors incurred by basis set and CI truncation.

\section{CONCLUSIONS}

An effective Hamiltonian based method is introduced which eliminates the main intruder state effects encountered in variational perturbation theory. The reference space is treated as quasidegenerate and the effects of single and double excitations from the reference space are obtained in an approximately size-consistent manner. QDVPT preserves the advantages of VPT in that changes in the reference space coefficients brought on by correlation are possible, unlike MRLCC. A novel method for solving the QDVPT equations is introduced that avoids explicit construction of the effective Hamiltonian over the reference space. As a result, QDVPT takes little more computational time than MRLCC or VPT.

Approximate expressions for the evaluation of one-electron properties for variational perturbation theory, quasidegenerate variational perturbation theory, and multireference linearized coupled-cluster wave functions are presented. Results are compared to truncated $\mathrm{CI}$ and full $\mathrm{CI}$ results and good agreement is obtained.

It is shown that the inclusion of the orthogonal complement of $\Psi_{0}$ in the variational perturbation theory wave function leads to size-inconsistency effects. These effects are relatively minor in the total energy, but can be quite large in the one-electron properties.

\section{ACKNOWLEDGMENTS}

The authors gratefully acknowledge financial support of this research by the National Institutes of Health, Grant No. 2 R01 GM34081-03. The calculations were performed in part at the Indiana University Computational Facility, the establishment of which was in part made possible by grants from the National Science Foundation, Grant Nos. CHE-83-09446 and CHE-84-05851.

\section{APPENDIX}

The direct solution of Eq. (21) via Eqs. (22) and (23) (i.e., formation of the effective Hamiltonian) requires approximately $n$ times the work of an MRLCC calculation, where $n$ is the number of spin-adapted configurations in the reference space. In this Appendix we introduce an iterative procedure for the solution of Eq. (21) which, for the calculations presented above, converged faster than the effective Hamiltonian method and becomes increasingly faster as $n$ increases.

We begin with Eq. (21) and partition it as one would in a VPT calculation. That is, we single out one function in the 
reference space (normally that associated with $E_{0}$, designated $\Psi_{0}^{i}$ ), write $\Psi$ in intermediate normalized form based on $\Psi_{0}^{i}$ and rewrite Eq. (21) as the two sets of equations:

$$
E=E_{0}+\sum_{j \neq i}\left\langle\Psi_{0}^{i}|H| C^{j}{ }^{j}, \Psi_{0}^{j}\right\rangle+\sum_{j}\left\langle\Psi_{0}^{i}|H| C_{Q}^{j} \phi_{j}\right\rangle
$$

and

$$
\left(\begin{array}{cc}
\mathbf{P}^{\prime} \mathbf{H} \mathbf{P}^{\prime}-\mathbf{E} & \mathbf{P}^{\prime} \mathbf{H Q} \\
\mathbf{Q H} \mathbf{P}^{\prime} & \mathbf{Q H Q}-\mathbf{E}_{\mathbf{0}}
\end{array}\right)\left(\begin{array}{l}
\mathbf{C}_{\mathbf{P}^{\prime}} \\
\mathbf{C}_{\mathbf{Q}}
\end{array}\right)=-\left(\begin{array}{c}
\mathbf{P}^{\prime} \mathbf{H} \Psi_{0}^{i} \\
\mathbf{Q H} \Psi_{0}^{i}
\end{array}\right)
$$

$\mathbf{P}^{\prime}$ represents the orthogonal complement of $\Psi_{0}^{i}$ in the reference space. Equations (A1) and (A2) are identical to Eqs. (22) and (23). They are also formally similar to the VPT equations, except that $E$ in the $\mathbf{P}^{\prime}$ block would be replaced by $E_{0}$. It is the presence of $E$ that prevents one from simply solving Eq. (A2) as a set of inhomogeneous linear equations.

Rather than constructing the effective Hamiltonian of Eq. (23) we replace $E$ in Eq. (A2) with an approximation to the total correlation energy and solve Eq. (A2) for approximate $C_{P^{\prime}}$ and $C_{Q}$. Using Eq. (A1) one obtains a new approximation to the total correlation energy based on these $C_{P^{\prime}}$ and $C_{Q}$. Iterating this procedure leads (when it converges) to results identical with the direct solution of Eq. (21) via Eqs. (22) and (23). This iterative method (with the modifications described below to speed convergence) is the one we used to obtain the QDVPT results in the present article, and it affords significant speedups over formation of the effective Hamiltonian, especially when the reference space is large. [Only for cases having two reference functions where the energy was converged to quite high accuracy (better than $1.0 E-10$ ) was the effective Hamiltonian method competitive in the present study. It may also be competitive for tworeference case where a particularly bad initial guess at $E$ is used or for near degeneracies.] After completion we renormalize $\Psi$ to have the proper normalization for property evaluation (i.e., $\Sigma\left|C_{P}\right|^{2}=1$ ).

One can go a step further to speed convergence, with no increase in work. The above iterative expression has first order errors in $E_{n+1}-E_{n}$; i.e., it converges linearly with the error in $E$. Below we develop an iterative procedure which eiliminates the errors linear in $E_{n+1}-E_{n}$ and obtain an approximately quadratically convergent method. The procedure is analogous to one used to obtain approximately quadratic convergence for the $B_{K}$ method. ${ }^{43}$

Using the subscript 0 to refer to $\Psi_{0}^{i}$, and with $\mathbf{P}^{\prime}$ and $\mathbf{Q}$ defined as for Eqs. (A1) and (A2) one can rewrite Eqs. (A1) and (A2) as

$$
\begin{aligned}
E= & H_{00}+H_{0 P^{\prime}}\left\{P^{\prime}\left(E-H_{P^{\prime} P^{\prime}}^{e}\right) P^{\prime}\right\}^{-1} H_{P^{\prime} 0}^{e} \\
& +H_{0 Q}\left\{Q\left(E_{0}-H_{Q Q}\right) Q\right\}^{-1} H_{Q 0}^{e}
\end{aligned}
$$

with

$$
\begin{aligned}
& H_{P^{\prime} P^{\prime}}^{e}=H_{P^{\prime} P^{\prime}}+H_{P^{\prime} Q^{\prime}}\left\{Q\left(E_{0}-H_{Q Q}\right) Q\right\}^{-1} H_{Q^{\prime}}, \\
& H_{P^{\prime} 0}^{e}=H_{P^{\prime} 0}+H_{P^{\prime} Q}\left\{Q\left(E_{0}-H_{Q Q}\right) Q\right\}^{-1} H_{Q^{0}}, \\
& H_{Q^{0}}^{e}=H_{Q^{\prime}}+H_{Q^{\prime}}\left\{P^{\prime}\left(E-H_{P^{\prime} P^{\prime}}^{e}\right) P^{\prime}\right\}^{-1} H_{P^{\prime} O}^{e}
\end{aligned}
$$

We assume $E_{n+1}$ is known and is essentially converged. Using Eq. (A3), which is satisfied when $E_{n+1}$ is the converged energy, we expand $\left(\mathrm{E}_{n+1}-H\right)^{-1}$ about $E_{n}$. [Note, in the iterative scheme $E_{n+1}$ is determined via $E_{n}$ using Eq. (A3).] Expansion of $\left\{P^{\prime}\left(E_{n+1}-H_{P^{\prime} p^{\prime}}^{c}\right) P^{\prime}\right\}^{-1}$ around $E_{n}$ yields [with $\Delta E_{n}=\left(E_{n+1}-E_{n}\right)$ ]

$$
\begin{aligned}
\left\{P^{\prime}\left(E_{n+1}-H_{P^{\prime} P^{\prime}}^{e}\right) P^{\prime}\right\}^{-1} \\
\simeq\left\{P^{\prime}\left(E_{n}-H_{P^{\prime} P^{\prime}}^{e}\right) P^{\prime}\right\}^{-1}+\left\{P^{\prime}\left(E_{n}-H_{P^{\prime} P^{\prime}}^{e}\right) P^{\prime}\right\}^{-1} \\
\quad \times\left(\Delta E_{n}\right)\left\{P^{\prime}\left(E_{n}-H_{P^{\prime} P^{\prime}}^{e}\right) P^{\prime}\right\}^{-1}+O\left[\left(\Delta E_{n}\right)^{2}\right] .
\end{aligned}
$$

Retaining only the first two terms and substituting back into Eq. (A3), noting that

$$
\begin{aligned}
C_{P^{\prime}}^{n+1}= & \left\{P^{\prime}\left(E_{n}-H_{P^{\prime} P^{\prime}}^{e}\right) P^{\prime}\right\}^{-1} \\
& \times\left\{H_{P^{\prime} 0}+H_{P^{\prime} Q^{\prime}}\left\{Q\left(E_{0}-H_{Q Q}\right) Q\right\}^{-1} H_{Q 0}\right\}
\end{aligned}
$$

one obtains the expression

$$
\begin{aligned}
E_{n+1}= & \left\{E_{0}+\sum_{j \neq i}\left\langle\Psi_{0}^{i}|H| C_{P}^{j}, \Psi_{0}^{j}\right\rangle\right. \\
& +\sum_{j}\left\langle\Psi_{0}^{j}|H| C_{Q}^{j} \phi_{j}\right\rangle \\
& \left.+E_{n} \Sigma\left|C_{P^{\prime}}\right|^{2}\right\} /\left(1+\Sigma\left|C_{P^{\prime}}\right|^{2}\right),
\end{aligned}
$$

where we take $C_{P}$, and $C_{Q}$ from the $(n+1)$ th iteration. This method yields approximately quadratic convergence for $E$.

Our method of solution proceeds as follows: (1) Guess an initial value for $E$, either based on Rayleigh-Schrödinger perturbation theory, size-consistency corrected $\mathrm{CI}$, or a previous MRLCC calculation; (2) solve Eq. (A2) using the method of Ref. 18; (3) use the result of (2) to construct Eq. (A9); (4) check for convergence. If the energy is not converged to the desired accuracy, repeat steps (2) and (3) with this new energy.

In tests on systems with larger reference spaces ${ }^{44}$ we have found that the present iterative scheme yields a workable procedure, whereas construction of the effective Hamiltonian becomes too time consuming to undertake.

'R. J. Bartlett, Annu. Rev. Phys. Chem. 32, 359 (1981).

${ }^{2}$ (a) C. W. Bauschlicher, S. R. Langhoff, P. R. Taylor, N. C. Handy, and P. J. Knowles, J. Chem. Phys. 85, 1469 (1986); (b) C. W. Bauschlicher and P. R. Taylor, ibid. 86, 1420 (1987); (c) 85, 6510 (1986); (d) Theor. Chim. Acta 71, 263 (1987).

${ }^{3}$ B. H. Brandow, Rey. Mod. Phys. 39, 771 (1967).

${ }^{4}$ S. Evangelisti, J. P. Daudey, and J.-P. Malrieu, Phys. Rev. A 35, 4930 (1987).

5. D. Laidig, P. Saxe, and R. J. Bartlett, J. Chem. Phys. 86, 887 (1987). 'W. D. Laidig and R. J. Bartlett, Chem. Phys. Lett. 104, 424 (1984).

'J. Paldus, in New Horizons in Quantum Chemistry, edited by P.-O. Lowdin and B. Pullman (Reidiel, Dordrecht, 1983), pp. 31-60.

${ }^{8}$ B. Jeziorski and J. Paldus, J. Chem. Phys. 88, 5673 (1988).

${ }^{9}$ H. Baker and M. A. Robb, Mol. Phys. 50, 1077 (1983).

${ }^{10} \mathrm{~K}$. Tanaka and H. Terashima, Chem. Phys. Lett. 106, 558 (1984).

"'(a) A. Banerjee and J. Simons, Int. J. Quantum Chem. 19, 207 (1981); (b) J. Chem. Phys. 76, 4548 (1982).

${ }^{12}$ M. R. Hoffmann and J. Simons, J. Chem. Phys. 88, 993 (1988).

${ }^{13}$ (a) M. A. Haque and D. Mukherjee, J. Chem. Phys. 80, 5058 (1984); (b) D. Mukherjee, Chem. Phys. Lett. 125, 207 (1986); (c) D. Sinha, S. K Mukhopadhyay, M. D. Prasad, and D. Mukherjee, ibid. 125, 213 (1986); (d) S. Pal, M. Rittby, R. J. Bartlett, D. Sinha, and D. Mukherjee, ibid. 137, 273 (1987); (e) D. Mukherjee, W. Kutzelnigg, and S. Koch, J. 
Chem. Phys. 87, 5902 (1987); (f) S. Pal, M. Rittby, R. J. Bartlett, D. Sinha, and D. Mukherjee, ibid. 88, 4357 (1988).

${ }^{14}$ U. Kaldor, J. Chem. Phys. 87, 467 (1987), and references cited therein.

${ }^{15}$ R. J. Gdanitz and R. Ahlrichs, Chem. Phys. Lett. 143, 413 (1988).

${ }^{16}$ E. A. Hylleraas, Z. Phys, 65, 209 (1930)

${ }^{17}$ H. A. Bethe and E. E. Salpeter, Quantum Mechanics of One- and TwoElectron Systems (Springer, Berlin, 1957), p. 122.

${ }^{1 K}$ R. J. Cave and E. R. Davidson, J. Chem. Phys. 88, 5770 (1988).

${ }^{19}$ D. Feller, C. M. Boyle, and E. R. Davidson, J. Chem. Phys. 86, 3424 (1987).

${ }^{20} \mathrm{H}$. Hellmann, Einfuehrung in Quantenchemie (Deuticke, Leipzig, 1937), p. 285; R. P. Feyman, Phys. Rev. 56, 340 (1939).

${ }^{21}$ A. C. Hurley, Proc. R. Soc. London Ser. A 226, 179 (1954)

${ }^{2}$ See A. Szabo and N. S. Ostlund, Modern Quantum Chemistry: Introduction to Advanced Electronic Structure Theory (MacMillan, New York, 1982).

${ }^{23}$ J. A. Pople, J. W. McIver, Jr., and N. S. Ostlund, J. Chem. Phys. 49, 2960 (1968).

${ }^{24}$ J. N. Franklin, Matrix Theory (Prentice-Hall, Englewood Cliffs, NJ, 1968), p. 22.

25 (a) P. Pulay and S. Saebo, Theor. Chim. Acta 69, 357 (1986); (b) K. Wolinski, H. I. Sellers, and P. Pulay, Chem. Phys. Lett. 140, 225 (1987).

${ }^{26}$ G. Hose and U. Kaldor, J. Phys. B 12, 3827 (1979).

${ }^{27}$ I. Lindgren, Int. J. Quantum Chem., Quantum Chem. Symp. 12, 33 (1978).

${ }^{2 \mathrm{~B}} \mathrm{~B}$. Jeziorski and H. J. Monkhorst, Phys. Rev. A 24, 1668 (1981).

${ }^{29} \mathrm{P}$.-O. Lowdin, in Perturbation Theory and its Applications in Quantum Mechanics, edited by C. H. Wilcox (Wiley, New York, 1966), p. 255.
${ }^{30} \mathrm{~W}$. Kutzenlnigg, Chem. Phys. Lett. 35, 283 (1975).

${ }^{3}$ 'Z. Gershgorn and I. Shavitt, Int. J. Quantum Chem. 2, 751 (1968).

${ }^{32}$ (a) E. R. Davidson, L. E. McMurchie, and S. J. Day, J. Chem. Phys. 74, 5491 (1981); (b) D. C. Rawlings and E. R. Davidson, Chem. Phys. Lett. 98, 424 (1983).

${ }^{33}$ The MELDF series of electronic structure codes was developed by $\mathbf{L}$. E. McMurchie, S. T. Elbert, S. R. Langhoff, E. R. Davidson, and D. Feller, and was extensively modified by $D$. C. Rawlings.

${ }^{34}$ G. D. Purvis III and R. J. Bartlett, J. Chem. Phys. 76, 1910 (1982).

${ }^{35}$ B. R. Brooks and H. F. Schaefer III, J. Chem. Phys. 68, 4839 (1978).

${ }^{36}$ G. D. Purvis III, R. Shepard, F. B. Brown, and R. J. Bartlett, Int. J. Quantum Chem. 23, 835 (1983).

${ }^{37}$ J. S. Binkley, J. A. Pople, and W. J. Hehre, J. Am. Chem. Soc. 102, 939 (1980).

${ }^{34}$ C. F. Bender and H. F. Schaefer III, J. Chem. Phys. 55, 4798 (1971).

${ }^{34}$ L. E. McMurchie and E. R. Davidson, J. Chem. Phys. 66, 2959 (1977).

${ }^{4 \prime}$ R. J. Cave and E. R. Davidson, J. Phys. Chem. 91, 4481 (1987).

${ }^{4}$ The dominant coefficients are defined as follows. The configurations were ordered on the basis of the size of the square of the coefficients in the final wave function. When two or more spin couplings exist for a given orbital occupation the sum of the squares of the coefficients for all spin couplings was used to order the configurations. Thus, it is perhaps more accurate to say that the dominant $n$-electron orbital products were selected, and all spin-adapted configuration state functions associated with them were included in the reference space.

${ }^{42}$ K. K. Sunil, K. D. Jordan, and R. Shepard, Chem. Phys. 88, 55 (1984).

${ }^{4.3}$ L. E. Nitzsche and E. R. Davidson, J. Chem. Phys. 68, 3103 (1978).

${ }^{44}$ D. Feller, R. J. Cave, and E. R. Davidson (unpublished results). 